\title{
LIE GROUPOIDS AS GENERALIZED ATLASES
}

\author{
JEAN PRADINES
}

\begin{abstract}
Starting with some motivating examples (classsical atlases for a manifold, space of leaves of a foliation, group orbits), we propose to view a Lie groupoid as a generalized atlas for the "virtual structure" of its orbit space, the equivalence between atlases being here the smooth Morita equivalence. This "structure" keeps memory of the isotropy groups and of the smoothness as well. To take the smoothness into account, we claim that we can go very far by retaining just a few formal properties of embeddings and surmersions, yielding a very polymorphous unifying theory. We suggest further developments.
\end{abstract}

\section{Contents}

1. Introduction.

2. Some motivating examples.

2.1. Regular equivalences.

2.2. Classical atlases.

2.3. Group and groupoid actions.

2.4. Foliations on $B$.

3. Diptychs.

3.1. Definition of diptychs.

3.2. Some variants for data and axioms.

3.3. A few examples of basic large diptychs.

3.4. (Pre)diptych structures on simplicial (and related) categories. 13

4. Commutative squares $\square$ in a diptych $\mathrm{D}=\left(\mathcal{D} ; \mathcal{D}_{i}, \mathcal{D}_{s}\right)$. 14

4.1. Three basic types of squares.

4.2. Basic diptych structures on $\square \mathcal{D}$.

5. Diagrams of type $T$ in a diptych. 16

5.1. Definitions : objects and morphisms. 17

5.2. Diptych structures on the category of diagrams.

5.3. The exponential law for diagrams. 18

6. Groupoids as diagrams in $\mathrm{E}=$ Set. 19

6.1. Some remarks about $\mathrm{N}_{c}^{+}$and $\mathrm{N}_{o}^{+}$. 19

6.2. Characterization of the nerve of a groupoid. 19

7. D-groupoids. 21

7.1. Definition of D-groupoids. $\quad 21$

\begin{tabular}{ll|} 
7.2. Principal and Godement D-groupoids. & 21 \\
\hline 7.3.
\end{tabular}

7.3. Regular groupoids. 23

8. The category $\operatorname{Gpd}(\mathrm{D})$. 24

Date: 04/11/03.

1991 Mathematics Subject Classification. 58H05.

Key words and phrases. Lie groupoids, spaces of leaves, orbit spaces. 
8.1. D-functors. 24

8.2. Actors. 24

8.3. D-equivalences. 25

8.4. Diptych structures on $\operatorname{Gpd}(\mathrm{D})$. 25

8.5. The category $\mathbf{G p d}\left(\mathrm{N}_{c}^{+*}\right)$. 26

9. Double functoriality of the definition of D-groupoids. 28

9.1. Bivariance of D-groupoids and D-functors. 28

9.2. Examples for the left functoriality. 29

9.3. Examples for the right functoriality. 30

9.4. D-natural transformations, holomorphisms.

10. The butterfly diagram. 31

10.1. Generalized "structure" of the orbit space. 31

10.2. Inverting equivalences. 31

10.3. Example.

11. Epilogue.

References

\section{INTRODUCTION.}

The aim of the present lecture is, rather than to present new results, to sketch some unifying concepts and general methods wished to be in Charles Ehresmann's spirit.

As usual I am expecting that geometers will think these sorts of concepts are too general and too abstract for being useful, while categoricists will estimate they are too special and too concrete for being interesting. However let us go.

In the following, I shall be concerned with a certain structure $B$ (basically thought as a manifold) endowed with a certain equivalence relation denoted by $\sim$ or $R$, and I would like to describe what kind of smoothness or structure is inherited from $B$ by the quotient set $Q=B / R$. The canonical projection will be denoted by $B \stackrel{q}{\rightarrow} Q$. The relation $R$ will be identified with its graph, defined by the following pullback square, in which $\beta=\operatorname{pr}_{1}, \alpha=\operatorname{pr}_{2}$. We also denote by $R \stackrel{\tau_{R}}{\rightarrow} B \times B$ the

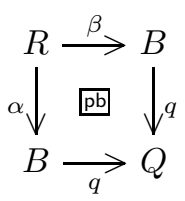

canonical injection, with $\tau_{R}=(\beta, \alpha)$.

In case when the structure $B$ is just a topology, the well known answer is given by the so-called quotient or identification topology on $Q$, which owns the good expected universal property in the category Top. However we notice that, when given other similar data $B^{\prime} \stackrel{q^{\prime}}{\rightarrow} Q^{\prime}$, one has not in general, in spite of a famous error (in Bourbaki's first edition), a homeomorphism between the product $Q \times Q^{\prime}$ and the quotient space of $B \times B^{\prime}$ by the product of the two equivalence relations, though this is true in two important cases, when $q$ and $q^{\prime}$ are both open or proper, since $q \times q^{\prime}$ has the same property. 
On the opposite, when $B$ is a manifold, it is well known that there is no such satisfactory answer when staying inside the category $\mathcal{D}=$ Dif of (smooth maps between) smooth manifolds, i.e. there is no suitable manifold structure for $Q$.

Now for facing this situation there may be two opposite, or better complementary, styles of approaches.

The first one consists in "completing" $\mathcal{D}$, i.e. embedding $\mathcal{D}$ in a larger category $\widehat{\mathcal{D}}$ by adding new objects in such a way than $\widehat{\mathcal{D}}$ has better categorical properties, i.e. has enough limits for allowing to define a good universal quotient. For instance one can wish $\widehat{\mathcal{D}}$ be a topos.

Various interesting solutions do exist, the study of which is out of our present scope. We just mention, besides Ehresmann's approaches, two dual ways (considered, under various aspects, by several lecturers at the present Conference) of defining generalized smooth structures on $Q$, one (first stressed by Frölicher) consisting in defining the smooth curves, while the second method (emphasized by Souriau with his diffeologies) considers the smooth functions on $Q$. Alain Connes' "non-commutative" approach is also related.

We follow here an opposite path, avoiding to add too many (necessarily pathological) new objects, and trying to stay within $\mathcal{D}$. We do not attempt to define a generalized smooth structure (in the set-theoretical sense) on the most general quotients, and limit ourself to objects which are sufficiently close to manifolds in the sense that they can be described by means of equivalence classes of some simple types of diagrams in $\mathcal{D}$; we do not try to introduce the limits of such diagrams in the categorical sense.

Indeed we think that the classical categorical concept of limit involves in general a certain loss of the information encapsulated in the concept of a suitable equivalence class of diagrams, but we shall not attempt to develop more formally such a general concept here, though we think it a very promising way, being content with illustrating this point of view by the important special case sketched presently.

\section{Some motivating EXAmples.}

Before going to abstract general definitions, I start by giving some elementary examples (to be made more precise later) of the kinds of objects I have in mind.

2.1. Regular equivalences. The ideal situation is of course that of the so-called regular equivalences. This means that there exists on $Q$ a (necessarily unique) manifold structure such that $B \stackrel{q}{\longrightarrow} Q$ is a surmersion (= surjective submersion). (Here we start anticipating some pieces of notation for arrows to be systematized later within a more general setting).

Godement's theorem gives a characterization of those equivalences by properties of the graph $R$ summarized by the following notations :

$$
R \underset{\beta}{\stackrel{\alpha}{\longrightarrow}} B \text { and } R \stackrel{\tau_{R}}{\longrightarrow} B \times B
$$

where again the black triangle head for an arrow stands for "surmersion", while the black triangle tail means "embedding" (in the sense of Bourbaki), or "proper embedding" when dealing with Hausdorff manifolds. 
These conditions express that $R$, regarded (in a seemingly pedantic way) as a subgroupoid of the (banal) groupoid $B \times B$, is indeed a smooth (or Lie) groupoid in the sense introduced by Ehresmann, embedded in $B \times B$, and the manifold $Q$ may be viewed as the orbit space of this groupoid.

A Lie groupoid $R$ satisfying the framed conditions will be called a principal or Godement groupoid.

We shall see in the next example why it is convenient to consider

$$
R \underset{\beta}{\stackrel{\alpha}{\longrightarrow}} B \stackrel{q}{\longrightarrow} Q
$$

as a "generalized (non étale) atlas" for $Q$.

If we have another "atlas" $R^{\prime} \underset{\beta}{\stackrel{\alpha}{\longrightarrow}} B^{\prime} \stackrel{q^{\prime}}{\longrightarrow} Q$ of the same manifold $Q$, we can take the fibred product of $q$ and $q^{\prime}$, and we get a commutative diagram express-

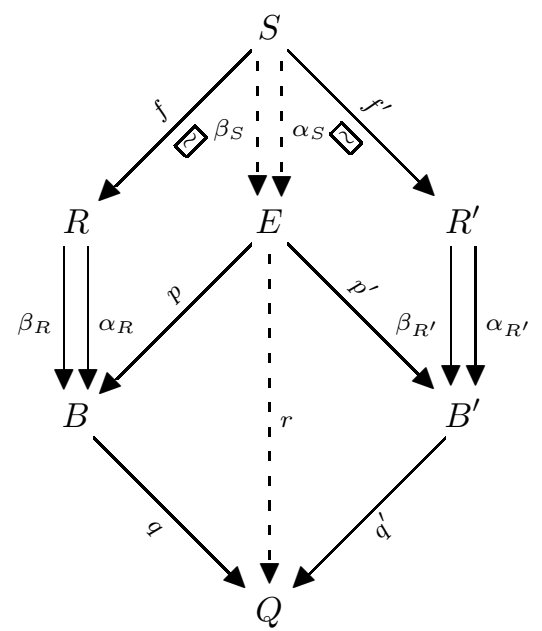

ing the "compatibility" of these "atlases", which means that they define the same (manifold) structure on $Q$.

More precisely the graph $S$ (which in turn may be viewed as a groupoid) can be obtained in the following way by means of the commutative cube below, the bottom

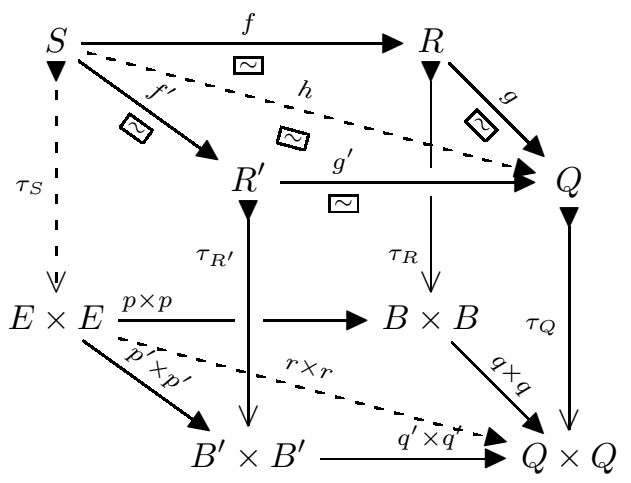

face of which is the pullback of $q \times q$ and $q^{\prime} \times q^{\prime}$, and which is constructed step by step by pulling back along the vertical arrows, starting with $\tau_{Q}$. The last one is just the diagonal of $Q$, and may be considered as the anchor map of the "null" groupoid 
$Q$ (consisting of just units). The upper face is then also a pullback, as well as all the six faces, and also the vertical diagonal square with three dashed edges. The $\sim$ symbols emphasize (very special instances of) "surmersive equivalences" between Lie groupoids. (One can observe on this diagram the general property of "parallel transfer by pulling back" for the embeddings and surmersions).

Thus we see that the "compatibility" of the two (generalized) atlases

$$
R \underset{\beta}{\stackrel{\alpha}{\longrightarrow}} B \stackrel{q}{\longrightarrow} Q \text { and } R^{\prime} \underset{\beta}{\stackrel{\alpha}{\longrightarrow}} B^{\prime} \stackrel{q^{\prime}}{\longrightarrow} Q
$$

for $Q$ is expressed by the existence of a common "refinement" (pictured above with dashed arrows) : $S \underset{\beta}{\stackrel{\alpha}{\longrightarrow}} E \stackrel{r}{\longrightarrow} Q$.

One might prove directly a converse, which indeed follows from more general considerations.

2.2. Classical atlases. An important special case of the previous one (which explains the terminology) will be given by the following diagrammatic description of atlases and covers of a manifold $Q$.

Let $\left(\varphi_{i}: V_{i} \rightarrow U_{i}\right)_{(i \in I)}$ be an atlas of the manifold $Q$, where $\left(V_{i}\right)_{(i \in I)}$ is a cover of $Q$ and the codomains $U_{i}$ 's of the charts $\varphi_{i}$ 's are open sets in some model space (which may be $\mathbb{R}^{n}$ or a Banach space).

Let $V_{i j}$ be $V_{i} \cap V_{j}$ and $U_{i j}$ be the image of $V_{i j}$ in $U_{i}$ by the restriction of $\varphi_{i}$.

Set $V=\coprod_{i \in I} V_{i}$, with its canonical projection $r: V \rightarrow Q$ (whose datum is equivalent to the datum of the covering), $U=\coprod_{i \in I} U_{i}$ (a trivial manifold), $R=$ $\coprod_{(i, j) \in I \times J} U_{i j}$, and $S=\coprod_{(i, j) \in I \times J} V_{i j}$. The charts $\varphi_{i}$ 's define a bijection $\varphi: V \rightarrow$ $U$ as well as a bijection $\phi: S \rightarrow R$.

Note that $S$, together with its canonical projection onto $Q$, defines the intersection covering, while, with its two canonical projections onto $V$, it can be viewed also as the graph of the equivalence relation associated to the surjection $r$.

Using the bijections $\varphi, \phi$, we have analogous considerations for $R$ and $U$, but moreover the latter are (trivial) manifolds, and the equivalence is regular, so that we recover a (very) special instance of the situation in the first example. Here the projections $\alpha_{R}, \beta_{R}$ are not only surmersions but moreover étale maps (of a special type, which might be called trivial) ; here they will be pictured by arrows of type $\longrightarrow$. More precisely their restrictions to the components of the coproduct $R$ define homeomorphisms onto the open sets $U_{i j}$ 's, and the datum of the smooth groupoid $R$ with base $U$ is precisely equivalent to the datum of the pseudogroup of changes of charts.

The situation is summed up by the following diagram (with $q=r \circ \varphi^{-1}$ ), which

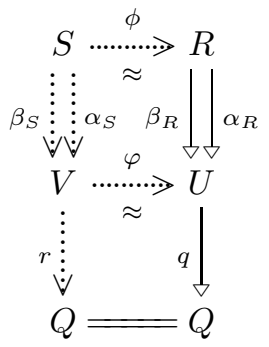


describes the generalized atlas $R \stackrel{\alpha}{\longrightarrow} U \stackrel{q}{\longrightarrow} Q$ associated to a classical atlas (whence the terminology). The dotted arrows in the diagram are to remind that the left column lies in Set, while the right column lies in Dif.

Now if we define a refinement of the previous atlas, denoted by $U \stackrel{q}{\longrightarrow} Q$ for brevity, as an atlas $W \stackrel{r}{\longrightarrow} Q$ such that $r$ admits a surjective 1 factorization $W \stackrel{p}{\longrightarrow} U$, it is easy to see that the compatibility of two atlases $U \stackrel{q}{\longrightarrow} Q$ and $U^{\prime} \stackrel{q^{\prime}}{\longrightarrow} Q$ may be expressed by the existence of a common refinement $W \stackrel{r}{\longrightarrow} Q$, and we get a special case of the notion of compatibility introduced in the previous subsection, where the general surmersions are replaced by (very special) surjective étale maps.

The compatibility diagram now reads as below, and this explains the terminology

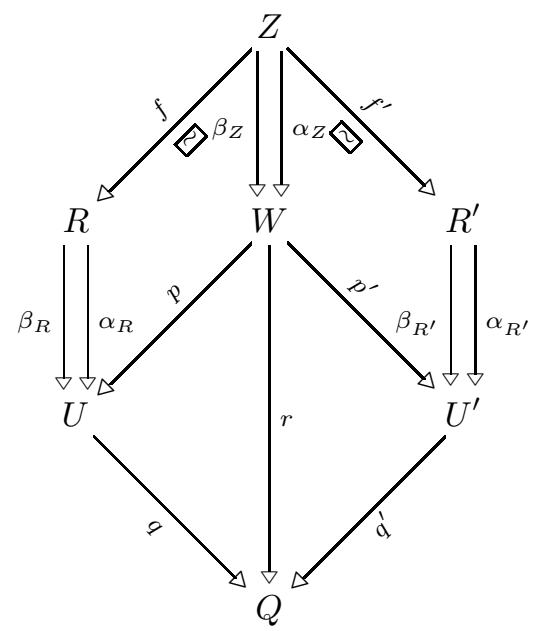

of the previous section.

2.3. Group and groupoid actions. An action of a Lie group $G$ on a manifold $E$, defined by the (smooth) map : $G \times E \stackrel{\beta}{\rightarrow} E,(g, x) \mapsto g \cdot x=\beta(g, x)$, can be described by the graph of the map $\beta$. Modifying the order in the products, this graph defines an embedding $H=G \times E \stackrel{\iota}{\longrightarrow} G \times(E \times E),(g, x) \mapsto(g,(g \cdot x, x))$. Regarding $G \times(E \times E)$ as a (Lie) groupoid with base $E$ (product of the group $G$ by the "banal" groupoid $E \times E$ ), the associativity property of the action law may be expressed by the fact that $H$ is an (embedded) subgroupoid of $G \times(E \times E)$. Composing $\iota$ with $\operatorname{pr}_{1}$ yields a (smooth) functor $H \stackrel{f}{\rightarrow} G$ which owns the property that the commutative diagram generated by the source projections is a pullback.

This construction extends for the action of a (smooth) groupoid $G \stackrel{\alpha}{\longrightarrow} B$ acting on a manifold over $B: E \stackrel{p}{\longrightarrow} B$, replacing the product $G \times(E \times E)$ by the

\footnotetext{
${ }^{1}$ This surjectivity is not implied by the usual definition of a refinement of a covering, but one can always impose it by the following slight modification of the definition, which changes nothing for the common use made of it : one demands in addition that a refinement of a covering contains this latter covering, which can always be achieved by taking their union.
} 
fibred product of the anchor map $\tau_{G}$ and $p \times p$, and one gets a pullback square :

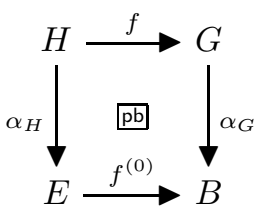

where $f^{(0)}=p$ (induced by the functor $f$ on the bases $\left.E=H^{(0)}, B=G^{(0)}\right)$. The action law may be recovered by $\beta=\beta_{H}$, using the isomorphism of $H$ with the fibred product of $G$ and $E$ over $B$, which results from the pullback property.

Note that the pullback property remains meaningful even when $E \stackrel{p}{\rightarrow} B$ is not a surmersion, since $\alpha_{G}$ still is, (though the fibred product of $\tau_{G}$ by $p \times p$ may then fail to exist), and $\beta_{H}$ still defines an action of $G$ on $p$.

For that reason we call a functor $H \underset{\text { act }}{\stackrel{f}{\longrightarrow}} G$ owning the previous pullback property an actor, which is emphasized by the framed label of the arrow. Such functors received various unfortunate names in the categorical literature, among which "discrete opfibrations" and "foncteurs d'hypermorphisme" (Ehresmann), and, better, "star-bijective" (Ronnie Brown), but note that the present concept encapsulates a smoothness information, included in the pullback property, and not only the purely set-theoretic or algebraic conditions (see below for a more general setting).

There is an equivalence of categories between the category of equivariant maps between action laws and the category admitting the actors as objects and commutative squares of functors as arrows.

Note than in the literature $H$ is currently called the action groupoid, but it is only the whole datum of the actor $f: H \rightarrow G$ which fully describes the action law, whence our terminology.

Here we let $Q$ be the (set-theoretic) quotient of the manifold $E$ by the action of the Lie group(oid) $G$. By the previous construction it appears too as the orbit space of the Lie groupoid $H$, so we have again for $Q=E / G=E / H$ a generalized atlas $H \underset{\beta}{\stackrel{\alpha}{\longrightarrow}} E \cdots \cdots$, the dotted arrow meaning here that we have now just a set-theoretic surjection (we have here to go out of Dif, since $Q$ is no more a manifold).

2.4. Foliations on $B$. Here we need a more restrictive notion for our surmersions, called retroconnected (it is in a certain sense precisely the opposite of being étale, which might be called as well "retrodiscrete"), and, in the present subsection, unlike the previous one, a notation such as $E \stackrel{p}{\longrightarrow} B$ will indicate that the surmersion $\mathrm{p}$ is retroconnected. This means that the inverse image of any $x \in B$ is connected, or, equivalently, that the inverse image of any connected subset of $B$ is connected.

In the following, when we have to use simultaneously étale and retroconnected surmersions, we shall distinguish them by means of circled labels :

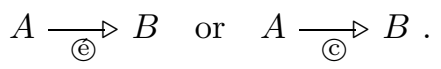

There is an obvious notion of foliation induced by pulling back a foliation along such a retroconnected surmersion, and the induced foliation keeps the same settheoretical space of leaves.

Then the notion of F-equivalence (in the sense introduced by $\mathrm{P}$. Molino in the 70 's) between two foliations $(B, \mathcal{F}),\left(B, \mathcal{F}^{\prime}\right)$ can be expressed by the existence of 
a commutative diagram as below, which means that $\mathcal{F}$ and $\mathcal{F}^{\prime}$ induce the same

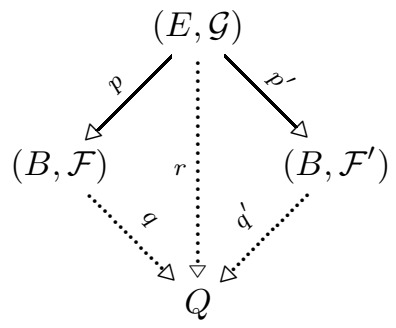

foliation $\mathcal{G}$ on the manifold $E$.

Now we note that again $Q=B / \mathcal{F}$ may be viewed as the orbit space of a Lie groupoid (here with connected source fibres), to know the Ehresmann holonomy groupoid $H \underset{\beta}{\stackrel{\alpha}{\longrightarrow}} B$ (sometimes renamed much later as the graph of the foliation), and Molino equivalence may alternatively be described by the commutative diagram below (with all maps retroconnected), where $f^{(0)}=p, f^{\prime(0)}=p^{\prime}$, and the symbols

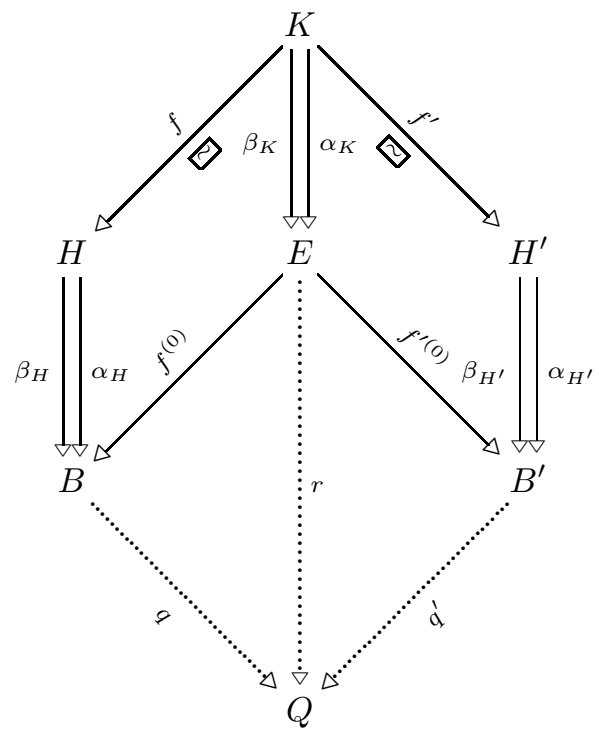

$\sim$ mean (surmersive retroconnected) equivalences of Lie groupoids, in a sense to be made more precise later.

As we shall see also later, such a diagram defines a (smooth in a very precise sense) Morita equivalence between $H$ and $H^{\prime}$.

Remark. Any surmersion $A \stackrel{f}{\longrightarrow} Q$ admits of an essentially unique2 factorization

$$
A \underset{\text { (C) }}{\stackrel{q^{\prime}}{\longrightarrow}} Q^{\prime} \underset{\text { (e) }}{\longrightarrow} Q
$$

with $q^{\prime}$ retroconnected and $e$ étale.

Though this might be proved directly, it is better to apply the general theory of Lie groupoids, in the special case of principal (or Godement) groupoids (see 2.1 above):

\footnotetext{
2 i.e. up to isomorphisms.
} 
if $R$ is the graph of the regular equivalence on $A$ defined by $q$, its neutral (or $\alpha$-connected) component $R^{c}$ is an open (possibly non closed3, but automatically invariant) subgroupoid of $R$, hence it is still a Godement groupoid, with now connected $\alpha$-fibres, and it defines the regular foliation admitting $Q^{\prime}$ f for its space of leaves. Then $Q^{\prime} \longrightarrow Q$ is a surmersion with discrete fibres, i.e. étale, and the graph of the equivalence on $Q^{\prime}$ thus defined is isomorphic to the two-sided quotient groupoid $R / / R^{c}$ (cf. [P2]).

\section{Diptychs.}

I am now enough motivated for introducing more dogmatically some general abstract definitions modelling and unifying the previous situations, as well as myriads of others.

3.1. Definition of diptychs. The notions presented in this section have a much wider range than it would be strictly necessary for the sequel, if one wants to stay in Dif, but give to it a much wider scope, even when aiming only at applications in Dif, as illustrated by some of the previous examples.

We introduced them a long time ago, in [P1], and think they deserve being better known and used.

In the presentation of the examples of the previous section, we emphasized the role played by embeddings/surmersions (these are special mono/epimorphisms of Dif, but not the most general ones, which would indeed be pathological), with possibly some more restrictive conditions added.

Our claim is that an incredible amount of various constructions can be performed without using the specificity of these conditions, but just a few very simple and apparently mild stability properties (of categorical nature) fulfilled in a surprisingly wide range of situations encountered by the "working mathematicians". The power of these properties comes from their conjunction.

Then the leading idea (illustrated beforehand in the previous section) will be to describe the set-theoretical constructions by means of diagrams, emphasizing injections/surjections, and then rereading these diagrams in the category involved, using the distinguished given mono/epi's.

3.1.1. Diptychs data. A "diptych" $\mathrm{D}=\left(\mathcal{D} ; \mathcal{D}_{i}, \mathcal{D}_{s}\right)$ (which may be sometimes denoted loosely by $\mathcal{D}$ alone) is defined by the following data :

- $\mathcal{D}$ is a category which comes equipped with finite non void 5 products.

The subgroupoid of invertible arrows (called isomorphisms) is denoted by $\mathcal{D}_{*}$.

- $\mathcal{D}_{i} / \mathcal{D}_{s}$ is a subcategory of $\mathcal{D}$, the arrows of which are mono/epi-morphisms (by axiom (iii) below), called good mono/epi's and denoted generally by arrows with a triangular tail/head such as $\longmapsto / \longrightarrow$, or $\longrightarrow / \longrightarrow$, and so on (here / is written loosely for resp.).

The arrows belonging to $\mathcal{D}_{r}=\mathcal{D}_{i} \mathcal{D}_{s}$ (i.e. composed of a good mono and a good epi) are called regular. In general $\mathcal{D}_{r}$ will not be a subcategory. When $\mathcal{D}_{r}=\mathcal{D}$, the diptych may be called regular.

\footnotetext{
${ }^{3}$ As in [B], we have to deal with possibly non Hausdorff manifolds.

${ }^{4}$ Possibly non Hausdorff.

${ }^{5}$ See below.
} 
3.1.2. Diptychs axioms. These data have to satisfy the following axioms (which look nearly self dual, but not fully, and that has to be noticed6) :

(i) $\mathcal{D}_{i} \cap \mathcal{D}_{s}=\mathcal{D}_{*}$;

(ii) $\mathcal{D}_{i}$ and $\mathcal{D}_{s}$ are stable by products ;

(iii) $\left(\right.$ a) $/(\mathrm{b})$ the arrows of $\mathcal{D}_{i} / \mathcal{D}_{s}$ are monos $/$ stric 7 epis ;

(iv) ( "strong/weak source/range -stability" of $\mathcal{D}_{i} / \mathcal{D}_{s}$ ) :

(a) $8\left(h=g f \in \mathcal{D}_{i}\right) \Rightarrow\left(f \in \mathcal{D}_{i}\right)$;

(b) $\left(\left(h=g f \in \mathcal{D}_{s}\right)\right.$ and $\left.\left(f \in \mathcal{D}_{s}\right)\right) \Rightarrow\left(g \in \mathcal{D}_{s}\right)$;

(v) ("transversality", denoted by $\mathcal{D}_{s} \pitchfork \mathcal{D}_{i}$ ):

(a) ("parallel transfer") :

given $A \stackrel{s}{\longrightarrow} B$ and $B^{\prime} \stackrel{i}{\longrightarrow} B$ (which means : $s \in \mathcal{D}_{s}, i \in \mathcal{D}_{i}$ ), there exists a pullback with moreover $s^{\prime} \in \mathcal{D}_{s}, i^{\prime} \in \mathcal{D}_{i}$ (the question marks

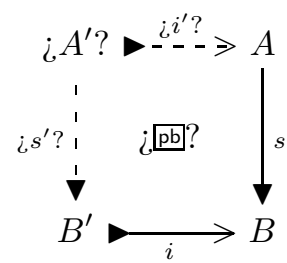

frame the objects or properties, as well as the dashed arrows, which appear in the conclusions, as consequences of the data).

(b) (conversely: "descent", or "reverse transfer") :

given a pullback square as below (with $i^{\prime} \in \mathcal{D}_{i}, s, s^{\prime} \in \mathcal{D}_{s}, i \in \mathcal{D}$ ),

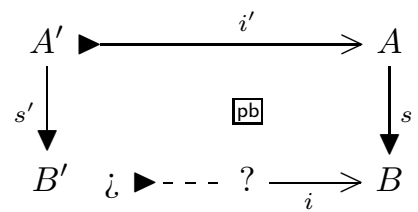

one has $i \in \mathcal{D}_{i}$ (conclusion pictured by the question marks around the dashed triangular tail).

3.1.3. Full subdiptychs. Remark. Let be given a subclass $\mathcal{C}$ of the class of objects of $\mathcal{D}$, which is stable by products and let $\mathcal{D}^{\prime}, \mathcal{D}_{i}^{\prime}, \mathcal{D}_{s}^{\prime}$ be the full subcategories of $\mathcal{D}$, $\mathcal{D}_{i}, \mathcal{D}_{s}$ thus generated.

In order they define a new dyptich $D^{\prime}$ (full subdiptych), one of the following conditions is sufficient :

(1) given a good epi $A \longrightarrow B$, the condition $B \in \mathcal{C}$ implies $A \in \mathcal{C}$;

(2) given a good mono $A \longmapsto B$, the condition $B \in \mathcal{C}$ implies $A \in \mathcal{C}$;

\subsection{Some variants for data and axioms.}

\footnotetext{
${ }^{6}$ It is said that the same thing happened at the very instant of the big bang, with analogous consequences.

${ }^{7}$ This means that they are coequalizers $[\mathrm{McL}]$. See below for an alternative formulation.

${ }^{8}$ We just mention that it may be sometimes useful to work with only the "weak source-stability" condition, dual of (b). It is then possible to define a suitable full subcategory of $\mathcal{D}$ in which the strong axiom is satisfied. The objects of this subcategory own (in particular) the property that their diagonal maps are in $\mathcal{D}_{i}$, which is formally a Hausdorff (or separation) type property. These objects may be called $i$-scattered.
} 
3.2.1. Prediptychs. When dealing with diagrams, we shall need a weaker notion: Definition. A prediptych is a triple $\mathrm{T}=\left(\mathcal{T} ; \mathcal{T}_{i}, \mathcal{T}_{s}\right)$ where one just demands $\mathcal{T}_{i}, \mathcal{T}_{s}$ to be subcategories of $\mathcal{T}$, such that :

$$
\mathcal{T}_{i} \cap \mathcal{T}_{s}=\mathcal{T}_{*} \text { (isomorphisms). }
$$

Most of the prediptychs we shall consider are regular, i.e. $\mathcal{T}=\mathcal{T}_{r}=\mathcal{T}_{i} \mathcal{T}_{s}$.

3.2.2. Alternatives for axioms. For any arrow $B \stackrel{f}{\longrightarrow} B^{\prime}$, we have the "graph factorization" : $B \stackrel{i}{\longrightarrow} B \times B^{\prime} \stackrel{\mathrm{pr}_{2}}{\longrightarrow} B^{\prime}$, with $i=\left(1_{B}, f\right) \in \mathcal{D}_{i}$ by axioms (iv) (a) and (i) (as a section of $\mathrm{pr}_{1}$ ), whence it is readily deduced that one has $\mathcal{D}_{s} \pitchfork \mathcal{D}$; this means :

- (v)(a) remains valid when omitting $\mathcal{D}_{i}$ both in assumptions and conclusions.

We have also $\mathcal{D}_{s} \pitchfork \mathcal{D}_{s}$. In particular the pullback square generated by two good epis $p, q$, has its four edges in $\mathcal{D}_{s}$. Such pullback squares will be called perfect squares. The case $p=q: B \longrightarrow Q$ covers the general situation embracing various examples above. It can be shown, using composition of pullback squares, that the axiom (iii) (b), in presence of the other ones, may be rephrased in the two following equivalent ways (using the notations of (2.10) :

- (iii) (b') any good epi $q: B \longrightarrow Q$ is the coequalizer of the pair $R \stackrel{\alpha}{\longrightarrow} B$, where $R$ is the fibred product of $q$ by $q$;

- (iii) (b") any perfect square is a push out too (this last property is very important and remarkable).

3.2.3. Terminal object. The existence of the void product (i.e. of a terminal object), is not always required, in view of important examples ; when it does exist, it will be denoted by a plain dot $\bullet$ (though its support has not to be a singleton).

Though, in many examples, not only there exists a terminal object, but moreover the canonical arrows $A \rightarrow \bullet$ are in $\mathcal{D}_{s}$, it may be useful however not to require this property in general. Then those objects owning this property will be called $s$-condensed (see examples below).

If $A \longrightarrow B$ is in $\mathcal{D}_{s}$, then $A$ is $s$-condensed iff $B$ is.

Observe that, if $A$ is $s$-condensed, then, for any object $Z$, the canonical projection $\operatorname{pr}_{2}: A \times Z \longrightarrow Z$ will be in $\mathcal{D}_{s}$ (which is always true whenever all objects are required to be $s$-condensed).

If $\mathcal{D}^{\prime}, \mathcal{D}_{i}^{\prime}, \mathcal{D}_{s}^{\prime}$ are the full subcategories generated by the s-condensed objects it can be checked that they define a (full) subdiptych $\mathrm{D}^{\prime}=\left(\mathcal{D}^{\prime} ; \mathcal{D}_{i}^{\prime}, \mathcal{D}_{s}^{\prime}\right)$.

3.3. A few examples of basic large diptychs. In fact most of the categories used by the "working mathematicians" own one or several natural diptych structures, and checking the axioms may sometimes be a more or less substantial (not always so well known) and often non trivial part of their theory, which is in this way encapsulated in the (powerful) statement that one gets a diptych structure 9

\footnotetext{
${ }^{9}$ It might be also advisable to look for a way of adapting the axioms without weakening their power in order to include some noteworthy exceptions such as measurable spaces or Riemannian or Poisson manifolds.
} 
This is all the more remarkable since a general category (with finite products) bears no canonical non trivial (i.e. with $\mathcal{D}_{s} \neq \mathcal{D}_{*}$ ) diptych structure, the crucial point being that in general the product of two epimorphisms fails to be an epimorphism.

3.3.1. Sets. The category $\mathcal{E}=$ Set of (applications between) sets owns a canonical diptych structure $\mathrm{E}=$ Set, which is regular, by taking for $\mathcal{E}_{i} / \mathcal{E}_{s}$ the subcategories of injections/surjections (here these are exactly all the mono/epi -morphisms).

The same is true for the dual category (exchanging injections and surjections), but the dual diptych $E^{*}$ is not isomorphic to $E$

3.3.2. Two general examples. There are two remarkable and important cases when one gets a (canonical) diptych structure, which is moreover regular, by taking as good monos/epis all the mono/epi -morphisms, to know : the abelian categories and the toposes 11 . Of course these two very general examples embrace in turn a huge lot of special cases in Algebra and Topology.

As a consequence all the constructions we carry out by using diptychs are working for general toposes, but the converse is false, since the most interesting and useful diptychs are not toposes.

3.3.3. Topological spaces. In Top (resp. Haus 12), we can take as good monos the (resp. proper 13 ) topological embeddings, and as good epis the surjective open maps. All objects are then $s$-condensed. This diptych is not regular.

These canonical diptychs will be denoted by Top and Haus.

We may alternatively take as good epis in Top the étale/retroconnected (or, in Haus, proper) surjective maps. Then the $s$-condensed objects are the discrete/connected (compact) spaces. This is illustrated by examples above.

3.3.4. Banach spaces. In Ban, the category of (continuous linear maps between) Banach spaces, one can take as good monos/epis the left/right invertible arrows. All objects are $s$-condensed. This diptych is not regular (save for the full subdiptych of finite dimensional spaces). It will be denoted by Ban.

3.3.5. Manifolds. In Dif, the category of (smooth maps between) possibly Banach and possibly non Hausdorff manifolds (in the sense of Bourbaki [B]), the basic diptych structure, denoted by Dif, is defined by taking as good monos the embeddings and as good epis the surmersions.

But ones gets a very large number of very useful variants and of full subdiptychs, as in Top, when suitably adding and combining extra conditions for objects or arrows such as being Hausdorff, proper, étale, retroconnected, and also various countability conditions, either on the manifolds (e.g. existence of a countable dense subset) or on the maps (for instance finiteness or countability of the fibres : retrofiniteness, retro-countability).

\footnotetext{
${ }^{10}$ Owing to the lack of symmetry for the axioms, one cannot in general define the dual of a diptych.

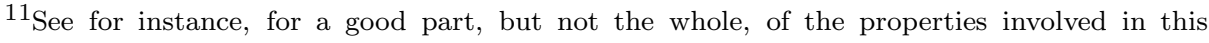
statement, the textbooks by Mac Lane and Peter Johnstone.

${ }^{12}$ The full subcategory of Hausdorff spaces.

${ }^{13}$ If we had taken these ones for Top, we would have been in the situation alluded to in footnote 8 and the Hausdorff spaces might be constructed as the i-scattered objects.
} 
This basic diptych is not regular 14 .

3.3.6. Vector bundles. Let VecB denote the category of (morphisms between) vector bundles (for instance in Dif) ; the arrows are commutative squares (see (4.1):

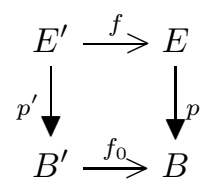

There are several useful diptych structures. The basic one, denoted by VecB, takes for good monos the squares with $f, f_{0} \in \mathcal{D}_{i}$, and for good epis those with $f, f_{0} \in \mathcal{D}_{s}$, and which moreover are $s$-full in the sense to be defined below (4.1). (One can also use pullback squares, which means $\mathrm{E}$ is induced by $B$ along $f_{0}$ ).

3.4. (Pre)diptych structures on simplicial (and related) categories. Small diptychs (and especially prediptychs) may be also of interest :

3.4.1. Finite cardinals. The category $\mathcal{N}_{c}$ of all maps between finite cardinals (or integers) defines a regular diptych $\mathrm{N}_{c}$ by taking for $\left(\mathcal{N}_{c}\right)_{i} /\left(\mathcal{N}_{c}\right)_{s}$ all the injections/surjections. The set of objects is $\mathbb{N}$. The $s$-condensed objects are those which are $\neq 0$.

The dual or opposite category, denoted by $\mathcal{N}_{c}^{*}$, defines also a regular diptych $\mathrm{N}_{c}^{*}$ with $\left(\mathcal{N}_{c}^{*}\right)_{i}=\left(\left(\mathcal{N}_{c}\right)_{s}\right)^{*},\left(\mathcal{N}_{c}^{*}\right)_{s}=\left(\left(\mathcal{N}_{c}\right)_{i}\right)^{*}$. The product in $\left(\mathcal{N}_{c}\right)^{*}$ is the sum in $\mathcal{N}_{c}$. The terminal object is now 0 , and all the objects are now $s$-condensed.

When dropping 0 one gets still diptychs denoted by $\mathrm{N}_{c}^{+}$and $\mathrm{N}_{c}^{+*} 15$, the objects of which will be denoted by $\cdot n=n+1(n \in \mathbb{N}) 16$.

The diptych $\mathrm{N}_{c}^{+*}$ will be basic for our diagrammatic description of groupoids 17 .

3.4.2. Finite ordinals. Considering now the integers as finite ordinals, we get the subcategories of monotone maps which will be denoted here by $\mathcal{N}_{o}, \mathcal{N}_{o}^{+}$, (denoted by $\Delta, \Delta^{+}$in $[\mathrm{McL}]$ ) (simplicial categories), as well as their duals, but these have no (cartesian) products and therefore cannot define diptychs. They define only prediptychs denoted here by : $\mathrm{N}_{o}, \mathrm{~N}_{o}^{+}, \mathrm{N}_{o}^{*}, \mathrm{~N}_{o}^{+*}$.

Remark. Though $\mathrm{N}_{o}$ is by no means isomorphic to its dual $\mathrm{N}_{o}^{*}$, however there are two canonical isomorphisms $\Phi, \Psi=\left(\Phi^{*}\right)^{-1}$ (defined only on the privileged subcategories!), which can be defined using the canonical generators as denoted in $[\mathrm{McL}]$ :

$$
\begin{aligned}
& \Phi:\left(\mathcal{N}_{o}\right)_{i} \rightarrow\left(\mathcal{N}_{o}^{*}\right)_{i}=\left(\left(\mathcal{N}_{o}\right)_{s}\right)^{*}, n \mapsto \cdot n, \delta_{j}^{n} \mapsto\left(\sigma_{j}^{\cdot n}\right)^{*}, \\
& \Psi:\left(\mathcal{N}_{o}\right)_{s} \rightarrow\left(\mathcal{N}_{o}^{*}\right)_{s}=\left(\left(\mathcal{N}_{o}\right)_{i}\right)^{*}, \cdot n \mapsto n, \sigma_{j}^{\cdot n} \mapsto\left(\delta_{j}^{n}\right)^{*},
\end{aligned}
$$

where a star bearing on an arrow means that this very arrow is regarded as belonging to the dual category (with source and target exchanged).

\footnotetext{
${ }^{14}$ This is indeed an important source of difficulty, but also of richness for the theory.

${ }^{15}$ The latter with a terminal object, the former without such.

${ }^{16}$ The notation suggests that the elements of such an object have to be numbered from 0 to $n$, the dot symbolizing the added 0 .

${ }^{17}$ We stress again that it is not isomorphic to $\mathrm{N}_{c}$.
} 
3.4.3. Canonical prediptychs. To each (small or not) category $\mathcal{T}$, we can associate three canonical prediptychs (the last two being regular):

$$
\mathrm{T}_{(*)}=\left(\mathcal{T} ; \mathcal{T}_{*}, \mathcal{T}_{*}\right), \mathrm{T}_{(\iota)}=\left(\mathcal{T} ; \mathcal{T}, \mathcal{T}_{*}\right) \text { and } \mathrm{T}_{(\sigma)}=\left(\mathcal{T} ; \mathcal{T}_{*}, \mathcal{T}\right) \text {. }
$$

3.4.4. Silly prediptychs. Let I (or sometimes, more pictorially, $\downarrow$ or $\rightarrow$ ) denote the (seemingly silly) category with two objects 0,1 , and one non unit arrow $0 \stackrel{\varepsilon}{\rightarrow} 1$ (it owns products and sums) 18 . It is canonically isomorphic with its dual $\mathrm{I}^{*}$, by exchanging the two objects.

All the arrows are both mono- and epi-morpisms, but $\varepsilon$ is not strict, and cannot be accepted as a good epi. As we shall see, it will be convenient to endow I with one of its canonical prediptych structures (3.4.3): $\mathrm{I}_{(*)}, \mathrm{I}_{(\iota)}, \mathrm{I}_{(\sigma)}$, according to what is needed.

\section{Commutative squares $\square$ in a Diptych $\mathrm{D}=\left(\mathcal{D} ; \mathcal{D}_{i}, \mathcal{D}_{s}\right)$.}

4.1. Three basic types of squares. Let $\square \mathcal{D} 19$ denote the category of commutative squares in $\mathcal{D}$ with the horizontal composition, which can be regarded (pedantically) as the category of natural transformations between functors from I to $\mathcal{D}$, with the (unfortunately so-called !) vertical composition. Its arrows might alternatively be described as functors from $I \times I$ to $\mathcal{D}$.

It turns out that the following properties of a commutative square $(K)$ play a

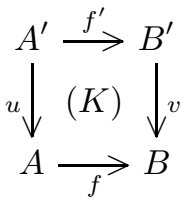

basic role (the terminology will be explained by the application to functors).

Definition : the commutative square $(K)$ is said to be :

. a) $i$-faithful if $A^{\prime} \stackrel{\left(u, f^{\prime}\right)}{\longrightarrow} A \times B^{\prime}$ is a good mono ; notation :

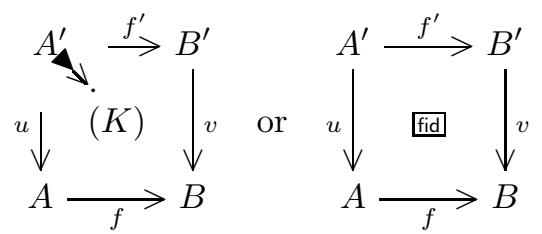

. b) a good pullback 20 if it is a pullback square (in the usual categorical sense) and moreover $i$-faithful 21 ; one writes $f \pitchfork v$ ( $f$ and $v$ are "weakly transversal") to mean that the pair $(f, v)$ can be completed in such a square ; notation :

\footnotetext{
${ }^{18}$ This is the category denoted by 2 in [McL], since it represents the order of the ordinal 2 .

${ }^{19}$ Ehresmann's notation.

${ }^{20}$ In Dif, there are plenty of useful pullback squares generated by pairs $(f, v)$ which are not transversal in the classical sense of [B] (for instance two curves intersecting neatly in a high dimensional manifold), but there are also (actually pathological) pullback squares existing without $(f, v)$ being weakly transversal. We think the weak transversality is the most useful notion.

${ }^{21}$ Observe that perfect squares are good pullbacks, as well as those arising from axiom (v), or more generally from (3.2.2), but it may happen that more general ones are needed.
} 


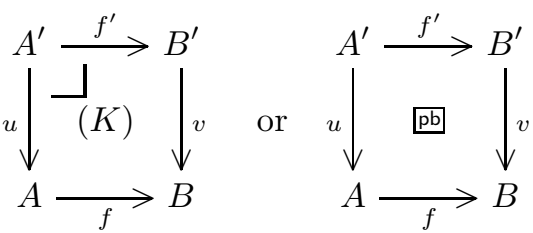

.c) $s$-full 22 if one has $f \pitchfork \sqrt{23}$ and if moreover the canonical arrow $A^{\prime} \longrightarrow A \times{ }_{B} B^{\prime}$ (which is then defined) is a good epi ; notation as below.

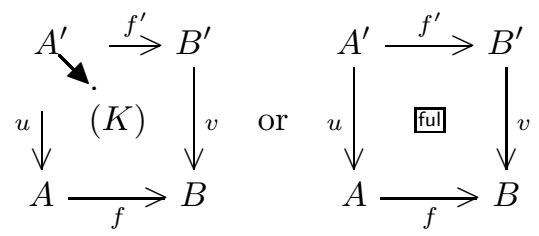

4.2. Basic diptych structures on $\square \mathcal{D}$. The three previous kinds of squares have remarkable composition stability properties resulting from the axioms, which we shall not state here (cf Prop. A 2 of [P3], with a different terminology). The (purely diagrammatical) proofs are never very hard, but may be lengthy.

A substantial part of these properties is expressed by the following important (non exhaustive) statements, which deserve to be considered as theorems, as they bring together a very large number of various properties, which acquire much power by being gathered.

Let be given a diptych $\mathrm{D}=\left(\mathcal{D} ; \mathcal{D}_{i}, \mathcal{D}_{s}\right)$.

4.2.1. Canonical structure. On $\square \mathcal{D}$, we get a first diptych structure, called canonical by setting :

$$
\square \mathrm{D}=\left(\square \mathcal{D} ; \square\left(\mathcal{D}, \mathcal{D}_{i}\right), \square\left(\mathcal{D}, \mathcal{D}_{s}\right)\right),
$$

which is pictured by :

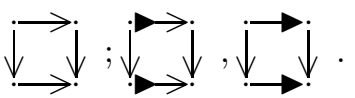

and also, using remark (3.1.3), the full subdiptych (still defined for the diptychs of the following paragraph):

$$
{ }_{i} \square \mathrm{D}=\left(\square\left(\mathcal{D}_{i}, \mathcal{D}\right) ; \square\left(\mathcal{D}_{i}, \mathcal{D}_{i}\right), \square\left(\mathcal{D}_{i}, \mathcal{D}_{s}\right)\right),
$$

pictured by :

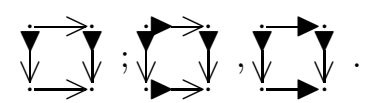

Defining an analogous full subdiptych with $\mathcal{D}_{s}$ replacing $\mathcal{D}_{i}$ requires a more restrictive choice for the squares taken as good epis. (These full subdiptychs will be essential to get fibred products of diagrams, hence of groupoids).

4.2.2. Full and pullback epis. We have the two basic diptychs:

$$
\begin{array}{|l|}
\square_{(\mathrm{i}, \text { ful })} \mathrm{D}=\left(\square \mathcal{D} ; \square\left(\mathcal{D}, \mathcal{D}_{i}\right), \text { 囬 }\left(\mathcal{D}, \mathcal{D}_{s}\right)\right) \\
\hline \hline \square_{(\mathrm{i}, \mathrm{pb})} \mathrm{D}=\left(\square \mathcal{D} ; \square\left(\mathcal{D}, \mathcal{D}_{i}\right), \text { 问 }\left(\mathcal{D}, \mathcal{D}_{s}\right)\right)
\end{array}
$$

which can be pictured by :

\footnotetext{
${ }^{22}$ Such a square owns the parallel transfer property $:\left(v \in \mathcal{D}_{s}\right) \Rightarrow\left(u \in \mathcal{D}_{s}\right)$.

${ }^{23}$ This was not demanded in a).
} 


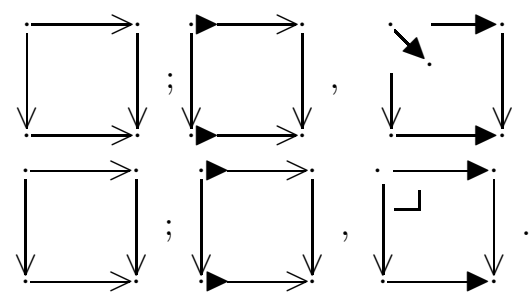

and in this way, thanks to a parallel transfer property, we get the expected basic full subdiptychs :

$$
\begin{array}{|l|}
{ }_{s} \square_{(\mathrm{i}, \text { ful })} \mathrm{D}=\left(\square\left(\mathcal{D}_{s}, \mathcal{D}\right) ; \square\left(\mathcal{D}_{s}, \mathcal{D}_{i}\right) \text {, fuul }\left(\mathcal{D}_{s}, \mathcal{D}_{s}\right)\right) \\
\hline \hline{ }_{s} \square_{(\mathrm{i}, \mathrm{pb})} \mathrm{D}=\left(\square\left(\mathcal{D}_{s}, \mathcal{D}\right) ; \square\left(\mathcal{D}_{s}, \mathcal{D}_{i}\right), \text { 回 }\left(\mathcal{D}_{s}, \mathcal{D}_{s}\right)\right)
\end{array}
$$

pictured by :

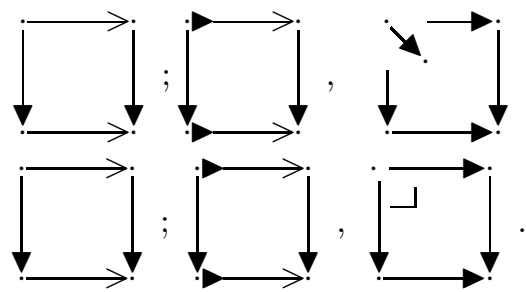

One also defines two basic subdiptychs by taking :

$$
\text { 四 } \mathrm{D}=\left(\text { 回 } \mathcal{D} ; \text { 问 }\left(\mathcal{D}, \mathcal{D}_{i}\right) \text {, 洄 }\left(\mathcal{D}, \mathcal{D}_{s}\right)\right)
$$

$$
s_{s} \text { 四 } \mathrm{D}=\left(\text { 四 }\left(\mathcal{D}_{s}, \mathcal{D}\right) \text {; 四 }\left(\mathcal{D}_{s}, \mathcal{D}_{i}\right) \text {, 四 }\left(\mathcal{D}_{s}, \mathcal{D}_{s}\right)\right)
$$

pictured by :

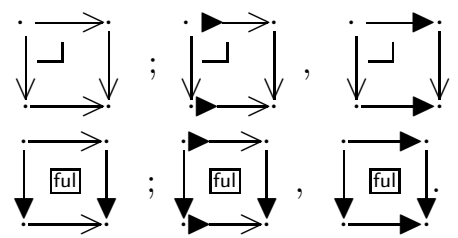

4.2.3. Iteration. One immediately notices than this theorem allows an iteration of the construction of commutative squares giving rise to new diptych structures for commutative cubes, and so on, which would be very difficult to handle directly.

This is especially interesting when dealing with commutative cubes since such a diagram gives rise to three commutative squares in $\square \mathcal{D}$, each edge of which (which is actually a square of $\mathcal{D}$ ) belonging to two different squares of $\square \mathcal{D}$, and this gives a powerful method for deducing properties of certain faces (for instance being a pullback), or edges from properties of the others, using diptych properties of parallel transfer. But we cannot develop more here.

\section{Diagrams of type T IN A DiPtych.}

We are now going to replace the silly category I of 3.4 .4 by a more general notion generalizing the construction of commutative squares, and allowing to perform various set-theoretic constructions in a general diptych $\mathrm{D}$. 
Let $\mathrm{T}$ be a small prediptych 24 (3.2.1), and let $\mathrm{D}=\left(\mathcal{D} ; \mathcal{D}_{i}, \mathcal{D}_{s}\right)$ be a diptych.

5.1. Definitions : objects and morphisms. We denote by $\square^{\mathcal{T}} \mathcal{D}:=\mathcal{D}^{\mathcal{T}}$ the category having :

- as objects the elements of $I^{\top} \mathrm{D}:=\operatorname{Hom}(\mathrm{T}, \mathrm{D})$, called diagrams of $\mathrm{D}$ of type $\mathrm{T}$, which means those functors $F$ from $\mathcal{T}$ to $\mathcal{D}$ such that one has :

$$
F\left(\mathcal{T}_{i}\right) \subset \mathcal{D}_{i} \text { and } F\left(\mathcal{T}_{s}\right) \subset \mathcal{D}_{s}
$$

(these last conditions are void when $\mathrm{T}=\mathrm{T}_{(*)}(3.4 .3)$ ) 25 ;

- as arrows the morphisms (i.e. the natural transformations) between such functor: 26 .

These morphisms may be described :

- either as functors $\Phi$ from $\mathcal{T} \times I$ to $\mathcal{D}$ such that (denoting by (:) the set of the two units of I):

$$
\Phi\left(\mathcal{T}_{i} \times(:)\right) \subset \mathcal{D}_{i} \text { and } \Phi\left(\mathcal{T}_{s} \times(:)\right) \subset \mathcal{D}_{s}
$$

- or as functors $\Psi$ from $\mathcal{T}$ to $\square^{\text {vert }} \mathcal{D} 27$ such that, with the notations of (4.2) :

$$
\Psi\left(\mathcal{T}_{i}\right) \subset \square\left(\mathcal{D}_{i}, \mathcal{D}\right)={ }_{i} \square \mathcal{D} \text { and } \Psi\left(\mathcal{T}_{s}\right) \subset \square\left(\mathcal{D}_{s}, \mathcal{D}\right)={ }_{s} \square \mathcal{D}
$$

In words this means that such a morphism may be viewed :

- either as a diagram in $\mathrm{D}$ of type $\mathrm{T} \times \mathrm{I}_{(*)}(\mathrm{cf} 3.4 .3$ and 3.4.4)

- or as a diagram of the same type $\mathrm{T}$ in $\square^{\mathrm{vert}} \mathrm{D}$, regarded with its canonical (vertical) diptych structure (4.2).

However be careful that, in such a description, though the previous definition of the morphisms uses the vertical composition of squares, the composition of these morphisms (called vertical in [McL]!!) involves the horizontal composition of squares.

\subsection{Diptych structures on the category of diagrams.}

5.2.1. Definitions. Using the latter interpretation, and taking now into account the horizontal composition, it is clear that any prediptych structure on $\square \mathcal{D}$ determines a prediptych structure on $\square^{\mathcal{T}} \mathcal{D}$. For instance, using on $T$ its trivial prediptych structure, we can consider the canonical prediptych structure $\square^{\top} \mathrm{D}$, but this not in general a dyptich structure (one needs parallel transfer properties for good epis in order to ensure conditions (1) of (5.1)).

Using the parallel transfer properties of $s$-full and pullback squares, one can get three useful diptych structures denoted by:

$$
\left(\square_{(\mathrm{i}, \mathrm{ful})}\right)^{\top} \mathrm{D},\left(\square_{(\mathrm{i}, \mathrm{pb})}\right)^{\top} \mathrm{D} \text { and } \mathrm{Bb}^{\top} \mathrm{D} \text {. }
$$

\footnotetext{
${ }^{24}$ It may be sometimes useful to extend the following definitions when $\mathrm{T}$ is just a graph with two given subgraphs.

${ }^{25}$ Forgetting the prediptych structure of $\mathrm{T}$ and dropping conditions (1) would oversimplify the theory, but deprive it of all its strength.

${ }^{26}$ Again with the "vertical composition", which we prefer here to write horizontally, drawing the diagrams vertically, and the morphisms horizontally. Of course one can exchange everywhere simultaneously "vertical" and "horizontal", since the distinction is purely psychological and notational, and since $\square^{\text {vert }} \mathcal{D}$ and $\square^{\text {hor }} \mathcal{D}$ are canonically isomorphic.

${ }^{27}$ This notation means that $\square \mathcal{D}$ has to be considered here with its vertical composition law.
} 
Of special interest, as we shall see, will be those diagrams which preserve certain pullbacks, since groupoids may be described by diagrams of this type.

5.2.2. The silly case. The case of commutative squares is just the special case when one takes for $\mathrm{T}$ the silly category $\mathrm{I}$, with one of its prediptych structures (3.4.4), since one has:

$$
\begin{gathered}
\mathrm{I}_{(*)} \mathcal{D}=|\mathcal{D}|, \mathrm{I}^{\mathrm{I}(\iota)} \mathcal{D}=\left|\mathcal{D}_{i}\right|, \mathrm{I}_{(\sigma)} \mathcal{D}=\left|\mathcal{D}_{s}\right|, 28 \\
\square^{\mathrm{I}(*)} \mathcal{D}=\square \mathcal{D}, \square^{\mathrm{I}(\iota)} \mathcal{D}={ }_{i} \square \mathcal{D} ; \square^{\mathrm{I}_{(\sigma)}} \mathcal{D}={ }_{s} \square \mathcal{D} ;
\end{gathered}
$$

more precisely :

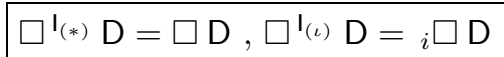

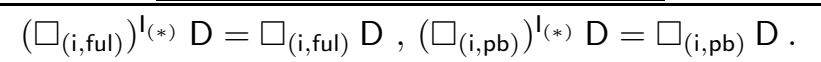

and also :

$$
\left({ }_{s} \square_{(\mathrm{i}, \mathrm{ful})}\right)^{\mathbf{l}_{(\sigma)}} \mathrm{D}={ }_{s} \square_{(\mathrm{i}, \mathrm{ful})} \mathrm{D},\left({ }_{s} \square_{(\mathrm{i}, \mathrm{pb})}\right)^{\mathbf{l}_{(\sigma)}} \mathrm{D}={ }_{s} \square_{(\mathrm{i}, \mathrm{pb})} \mathrm{D} .
$$

5.3. The exponential law for diagrams. Given another similar datum $\mathrm{S}$, we have the exponential law, which, with our notations, reads (at least when $\mathrm{S}$ and $\mathrm{T}$ are trivial diptychs) :

$$
\square^{\mathrm{S}}\left(\square^{\mathrm{T}} \mathrm{D}\right)=\square^{\mathrm{S} \times \mathrm{T}} \mathrm{D}
$$

(with a lot of possible variants), and the canonical isomorphism : $\mathrm{S} \times \mathrm{T} \approx \mathrm{T} \times \mathrm{S}$ yields a canonical isomorphism :

$$
\square^{\mathrm{S}}\left(\square^{\mathrm{T}} \mathrm{D}\right) \approx \square^{\mathrm{T}}\left(\square^{\mathrm{S} D}\right) \text {. }
$$

Particularly one has: $(\square \mathrm{T})^{n}=\square^{T^{n}}$,

and, specializing for $\mathrm{T}=\mathrm{I}_{(*)}:(\square)^{n}=\square^{\left(\mathrm{I}_{(*)}\right)^{n}}$.

Remarks about notations. The reader may have observed that the symbols $\square$ and I used above are intendedly ambiguous and protean, since this allows to memorize and visualize a lot of various properties :

- the symbols I or $\mathrm{I}_{(\bullet)}$, where $\bullet$ stands for $*, \iota$, or $\sigma$ :

- denote the silly category, possibly with various prediptych structures on it;

- denote the bifunctor Hom (?,?), with the first argument treated as an exponent);

- often behaves formally as a 1;

- may sometimes suggest the functor $\mathrm{T} \mapsto \mathrm{T} \times \mathrm{I}_{(\bullet)}$;

- the symbol $\square$ with possibly labels in various positions $\diamond \square{ }_{(\sharp, \natural)}^{\top}$ :

- may picture or suggest the product $I \times I$ or more precisely various instances of $\mathrm{I}_{(\triangle)} \times \mathrm{I}_{(\nabla)}$

- may create the commutative squares of a category or of a diptych with possible extra conditions :

* on the left, they bear on the vertical edges ;

* on the right, they describe the subcategories for a (pre)diptych structure ;

* inside, they describe global properties of the squares ;

- may become the bifunctor $\mathcal{H}$ om (?,?), with possible restrictions on the squares involved.

\footnotetext{
${ }^{28}$ In the second members of this line, the signs || denote the forgetful functor forgetting the composition laws, since they are not defined by the first members.
} 


\section{Groupoids as diagrams in $\mathrm{E}=$ Set.}

We shall be concerned only with small groupoids, viewed as generalizing both groups and graphs of equivalence relations, as well as specializing (small) categories.

According to our program, we need a diagrammatic description of both groupoid data and axioms, which will be transferred from the diptych $E=$ Set to a general diptych D.

This can be achieved in two complementary ways :

- either using finite diagrams (called sketches in Ehresmann's terminology) ;

- or using the simplicial description by the nerve.

Though seemingly more abstract, the latter turns out to be often the most convenient for theoretical purposes, while the former is adapted to practical handling.

We refer to [McL] and also to (3.4) for general properties and notations concerning simplicial categories and simplicial objects, which are just functors from $\mathcal{N}_{o}^{+*}$ to $\mathcal{E}$, and more precisely diagrams of type $\mathrm{N}_{o}^{+*}$ in $\mathrm{E}$.

\subsection{Some remarks about $\mathrm{N}_{c}^{+}$and $\mathrm{N}_{o}^{+}$. (see 3.4)}

6.1.1. The arrows as functors. For each $n \in \mathbb{N}$, we shall denote by :

$* \cdot \vec{n}$ the ordinal $\cdot n=n+1$ viewed as a small category (defining the order) 29;

$* \dot{\wedge} n$ the subcategory consisting of arrows of this order category with source 30 ;

$* \overleftrightarrow{n}=(\cdot n)^{2}$ the banal groupoid $(\cdot n) \times(\cdot n)$, which ignores the ordering 31 .

With these structures on the objects, the arrows of $\mathcal{N}_{o}^{+}$and $\mathcal{N}_{c}^{+}$may be regarded (though this looks pedantic) as functors (or morphisms) between small categories.

It may be sometimes suggestive to write :

$$
\overleftrightarrow{\cdot 0}=\overleftrightarrow{1}=\cdot, \overleftrightarrow{\cdot 1}=\overleftrightarrow{2}=\leftrightarrow, \dot{\wedge} 2=\wedge, \overleftrightarrow{2}=\overleftrightarrow{3}=\Delta, \overleftrightarrow{3}=\overleftrightarrow{4}=\bigotimes
$$

6.1.2. Pullbacks in $\mathcal{N}_{c}^{*}$. Pullbacks in $\mathcal{N}_{c}^{*}$ come from pushouts in $\mathcal{N}_{c}$. One can check that all the commutative squares describing the relations between the canonical generators of $\mathcal{N}_{o}$ (see [McL]) become pushouts when written in $\mathcal{N}_{c}$, and they generate, by composition, all the pushouts of $\mathcal{N}_{c}$. More precisely (with the notations in [McL]) the squares involving the injections $\delta_{j}$ 's alone or mixing both $\delta_{j}$ 's and $\sigma_{k}$ 's are pullbacks too, but not those with the surjections $\sigma_{j}$ 's alone 32 .

This is still valid when dropping 0 (but one looses the squares describing the sums as pushouts).

6.2. Characterization of the nerve of a groupoid. The special properties of a groupoid among categories yield very special and remarkable properties as well as alternative descriptions for its nerve.

6.2.1. Three descriptions for the nerve. Given a (small) groupoid $\mathbf{G}$, denoted loosely by $G$ or $G \rightrightarrows B$, we can associate to it three canonically isomorphic simplicial objects (among which the first one is the nerve of $G$ regarded as a category). First we define and denote the three images of the generic object $\cdot n=n+133$ :

(1) ${ }^{\downarrow} G^{(n)}=\operatorname{hom}(\overrightarrow{\cdot n}, G)=$ paths of $G$ of length $\left.n\right\}$;

$29 \overrightarrow{1}=\overrightarrow{2}$ is just what we called in 3.4 .4 the silly category $\mathrm{I}=\downarrow=\rightarrow$.

${ }^{30}$ And of course the units.

${ }^{31}$ Though the numbering of the base is kept!

${ }^{32}$ This induces a strong dissymmetry between the dual diptychs $\mathrm{N}_{c}$ and $\mathrm{N}_{c}^{*}$.

${ }^{33}$ Though these images may be regarded as instances of diagrams of finite type in $G$, the notations used below differ slightly from those used in the previous section for the general case. 
(2) ${ }^{\wedge} G^{(n)}=\operatorname{hom}(\dot{\wedge} n, G)=\{n$-uples of arrows of $G$ with the same source $\}$;

(3) ${ }^{\mathfrak{}} G^{(n)}=\operatorname{hom}(\overleftrightarrow{\cdot n}, G)=$ \{commutative diagrams of $G$ with $\cdot n$ vertices 3

Identifying these three sets, we can write loosely:

$$
G^{(n)}={ }^{\downarrow} G^{(n)}={ }^{\wedge} G^{(n)}={ }^{\downarrow} G^{(n)} .
$$

We shall also sometimes feel free to write loosely and suggestively :

$$
G^{(0)}=B, G^{(1)}={ }^{\downarrow} G={ }^{\downarrow} G=G, G^{(2)}=\wedge G=\triangle G, G^{(3)}=\otimes G .
$$

The images of the canonical generators of $\mathcal{N}_{c}^{+}$are then especially easy to define with the interpretation (3), since they consist in repeating or forgetting one vertex.

But, with the descriptions (1) and (3), one can immediately interpret the required contravariant functors from $\mathcal{N}_{o}^{+}$to $\mathcal{E}$ as being just special instances of the classical contravariant hom-functors hom(?, $G$ ), which consist in letting the arrows of $\mathcal{N}_{o}^{+}$act by right morphism composition (in the category of morphisms or functors between small categories) with the elements of ${ }^{\downarrow} G^{(n)}$ or ${ }^{\uparrow} G^{(n)}$.

\subsubsection{Properties of the nerve of a groupoid.}

. - (Extension of the nerve). With the interpretation (3), we get a bonus, since it is now obvious that this contravariant functor extends to $\mathcal{N}_{c}^{+}$, and so defines a diagram in $G$ of type $\mathrm{N}_{c}^{+*}$. (We remind that, in this interpretation, integers are interpreted as small banal groupoids, and the arrows of $\mathcal{N}_{c}^{+}$as morphisms.)

. - (Exactness properties). Moreover this extended functor, which is now defined on a diptych, is "exact", there meaning that it preserves pullbacks 35 . It would be enough to check this for the generating pullbacks of $\mathcal{N}_{c}^{*}$ (6.1.2).

- - Conversely one might check that the datum of an exact diptych morphism $\mathbf{G}=$ $\left(G^{(n)}\right)_{(n \in \mathbb{N})} 36$ from $\mathrm{N}_{c}^{+*}$ to $\mathrm{E}=$ Set determines a groupoid, the nerve of which is the restriction of this morphism to $\mathrm{N}_{o}^{+*}$.

. - (Sketch of a groupoid).37 Actually one might check that the groupoid data are fully determined by the restriction of $\mathbf{G}$ to the (truncated) full subcategory $[\cdot \mathbf{2}]$ of $\mathcal{N}_{c}^{+*}$ generated by $\cdot 0, \cdot 1, \cdot 2$, while the groupoid axioms are expressed by its restriction to $[\cdot \mathbf{3}]$ (and the conditions that the images of the previous generating squares be pullbacks) 38 .

6.2.3. Concrete description. Actually the previous data are somewhat redundant, and it is convenient for our purpose to observe that $\mathbf{G}$ may be fully described by the data $\left(G, B, \omega_{G}, \alpha_{G}, \delta_{G}\right)$ (satisfying axioms which we shall not make explicit), where :

- $B$ ("base") and $G$ ("set of arrows") are objects of $\mathcal{E}$;

- $\omega_{G}: B \longmapsto G$ is an injection ("unit law");

- $\alpha_{G}: G \longrightarrow B$ is a surjection (source map);

\footnotetext{
${ }^{34}$ With two-sided edges and numbered vertices.

${ }^{35}$ But not products (and not the pushouts which don't arise from perfect squares). When some risk of confusion might arise, it would be more correct to say something like "diptych-exact" or "p.b.-exact", since here the term "exact" has to be understood in a much weaker sense than the general meaning for functors between categories : it has not to preserve all (co)limits.

${ }^{36}$ Relaxed notation, using only the images of the objects.

37 This notion was introduced by Ehresmann for general categories.

${ }^{38}$ We cannot give more details here.
} 
- $\delta_{G}: \wedge G=\triangle G \longrightarrow G$ is a surjection ("division map" $\wedge G=G \times{ }_{B} G$ (fibred product of $\alpha$ by $\alpha$ ).

One often writes $\alpha$ for $\omega \alpha$, and we set :

- $\tau_{G}=\left(\beta_{G}, \alpha_{G}\right): G \rightarrow B \times B \quad$ ("anchor map" 40, or "transitor").

From these data, it is not difficult to get the range map $\beta_{G}$, the inverse law $\iota_{G}$, and the composition law $\gamma_{G}$ (defined on the fibred product of $\alpha_{G}$ and $\beta_{G}$ ).

With notations from Mac Lane: $\omega_{G}, \alpha_{G}, \beta_{G}, \delta_{G}$ would be the respective images of : $\left(\sigma_{0}^{1}\right)^{*},\left(\delta_{1}^{1}\right)^{*},\left(\delta_{0}^{1}\right)^{*},\left(\delta_{0}^{2}\right)^{*}$ ( in the dual $\left.\mathrm{N}_{c}^{*}\right)$.

\section{D-GROUPOIDS}

\subsection{Definition of D-groupoids.}

7.1.1. Groupoids in a diptych. The diagrammatic description of a set-theoretical groupoid given above leads to define a D-groupoid as an exact diptych morhism (6.2.2), i.e. preserving good monos, good epis, and good pullbacks:

$$
\mathbf{G}: \mathrm{N}_{c}^{+*} \rightarrow \mathrm{D} .
$$

As above, the exactness properties allow to characterize $\mathbf{G}$ by restriction to various subcategories, and to recover in this way the simplicial description as well as the skecth ones and the $\left(G, B, \omega_{G}, \alpha_{G}, \delta_{G}\right)$ presentation.

We shall also use as previously the relaxed notations $\left(G^{(n)}\right)_{n \in \mathbb{N}}$ (omitting the effect of $\mathbf{G}$ on the arrows), or briefly $G$ (meaning $\left.G^{(1)}\right), B$ for $G^{(0)}$, and $G \stackrel{\alpha}{\longrightarrow} B$ and its variants.

We shall denote by $\operatorname{Gpd}(\mathrm{D})$ the class of D-groupoids.

7.1.2. Examples. This very general notion may be specialized using for instance the various examples given in (3.3).

Note that the Top-groupoids are those for which the source map has to be open, a condition which can hardly be avoided for getting a useful theory.

Lie groupoids 41 are of course the Dif-groupoids, with the usual diptych structure on Dif, but, using some of the above-mentioned variants, the theory will include, for instance, among others, étale or $\alpha$-connected groupoids as well.

VecB-groupoids were used in [P6].

7.1.3. Null groupoids. For any object $B$ of $\mathcal{D}$, the constant simplicial object $B$ is a D-groupoid, called " $n u l l " 42$, and denoted by ${ }^{\circ} B$. All arrows are units, and the map $\omega_{G}$ is an isomorphism.

\subsection{Principal and Godement D-groupoids.}

\footnotetext{
${ }^{39}$ This means the map : $(y, x) \mapsto y x^{-1}$.

${ }^{40}$ Mackenzie's terminology.

${ }^{41}$ Introduced by C. Ehresmann under the name of differentiable groupoids.

${ }^{42}$ We cannot accept the traditional terminology "discrete", which has another topological meaning, and which moreover does not agree with the group terminology, unlike ours.
} 
7.2.1. The transitor $\tau_{G}$. From a purely set-theoretical point of view, the transitor $\tau_{G}: G \rightarrow B \times B$ measures the (in)transitivity 43 of the groupoid $G$.

It turns out that, in the diptych setting, its properties encapsulate a very rich "structured" information. We just mention, without developing here, that, for instance in Dif, one can fully characterize, just by very simple properties of $\tau_{G}$, not only the graphs of regular equivalences, but (among others) the gauge groupoids of principal bundles, the Poincaré groupoids of Galois coverings, the holonomy groupoids of foliations, the Barre Q-manifolds, the Satake V-manifolds (or orbifolds).

7.2.2. Principal D-groupoids. The notion of graph of regular equivalence (2.1) may be carried over in any diptych as follows.

Given a good epi $B \stackrel{q}{\longrightarrow} Q$ of $\mathrm{D}$, we can construct the iterated fibred product of $q$, denoted by $R^{(n)}={ }_{\times_{Q}}^{\cdot n} B(n \in \mathbb{N})$ and check that $\left(n \mapsto R^{(n)}\right)_{(n \in \mathbb{N})}$ allows to define a D-simplicial object which is a groupoid. We shall say that $\mathbf{R}=\left(R^{(n)}\right)_{n \in \mathbb{N}}$ is the principal groupoid associated to $q$ (with base $B$ ).

Moreover we have an "augmentation", which means an extended "exact" (6.2.2) diptych morphism (6.2.2) from $\mathrm{N}_{c}^{*}$ to $\mathrm{D}$. This augmentation carries 044 to $R^{(-1)}=$ $Q$, and the added generator $\eta^{*}=\left(\delta_{0}^{0}\right) * 45$ to $B \stackrel{q}{\longrightarrow} Q$.

This situation gives rise to a perfect square (3.2.2):

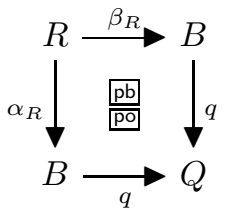

The pushout property of this square shows that the good epi $q$ is uniquely determined by the knowledge of the D-groupoid $R$.

Applied to $G \stackrel{\alpha_{G}}{\longrightarrow} B$, this construction allows considering $\wedge G$ as a principal groupoid (see below (8.2.2)).

7.2.3. Banal groupoids. This construction applies in particular when $B$ is an $s$ condensed object (3.2.3). Then one has $R^{(n)}=B^{\cdot n}$ ( $\cdot n$ times iterated product). It is called the "banal groupoid" 46 associated to $B$; it is principal. It is denoted by $B \times B$ or $B^{2}$.

More generally such a banal groupoid (possibly non principal in the absence of a terminal object) is associated to an object $B$ whenever the canonical projections $\operatorname{pr}_{i}: B \times B \rightarrow B$ are good epis 47 .

One might call "proper" those objects $B$ for which the banal groupoid $B^{2}$ is defined. When such is the case, the transitor $\tau_{G}$ may be viewed as a D-functor.

Given an integer $n$, we can attach to it, besides (for $n \neq 0$ ) the banal groupoid $\overleftrightarrow{n}=n^{2}$ (which uses the product in $\mathcal{N}_{c}$ ), a banal cogroupoid ${ }^{2} n$ or $2 n$, using the product $\times^{*}$ of $\mathcal{N}_{c}^{*}$ (which is the coproduct + of $\mathcal{N}_{c}$ ).

\footnotetext{
${ }^{43}$ Whence the terminology adopted here, preferably to "anchor map".

${ }^{44}$ Which might be written $0=\cdot(-1)$.

${ }^{45}$ Dual arrow, from Mac Lane's notations.

${ }^{46}$ We cannot accept the term "coarse", often used in the literature for the same reason as for "discrete".

${ }^{47}$ We gave above examples of diptychs in which such is not always the case.
} 
7.2.4. Godement groupoids and Godement diptychs. Definition. A D-groupoid $G$ is called a Godement groupoid if $\tau_{G}: G \longrightarrow B \times B$ is a good mono.

Every principal groupoid is a Godement groupoid.

- We say the Godement axiom is fulfilled, and D is a Godement diptych if conversely every Godement D-groupoid is principal.

The fact that the diptych Dif (3.3.5) is Godement is the content of the so-called Godement theorem, proved in Serre [LALG] 48 .

But it is highly remarkable that nearly all of the examples of diptychs given above are indeed Godement diptychs, as well as most of the diagram diptychs we constructed above, provided one starts with a Godement diptych. Such a statement includes a long list of theorems, which are not always classical.

- From now on, we shall assume $\mathrm{D}$ is Godement whenever this is useful.

7.3. Regular groupoids. More generally, a D-groupoid $G$ is called regular if $\tau_{G}$ is regular (3.1.1). Then we have the following factorization of $\tau_{G}$ :

$$
G \stackrel{\pi}{\longrightarrow} R \stackrel{\tau_{R}}{\longrightarrow} B \times B,
$$

where $\mathrm{R}$ is a Godement $\mathrm{D}$-groupoid, hence principal, so that we can construct the perfect square (7.2.2) ; then the orbit space $Q$ exists as an object of $\mathrm{D}$, and is also the pushout of $\alpha_{G}$ and $\beta_{G}$.

However the object $Q$ inherits an "extra structure" from the arrow $G \stackrel{\pi}{\longrightarrow} R$, a good epi which measures how much the s-full pushout square $G B B Q$, fails to be a pullback.

- In the general case, when $G$ is neither principal nor even regular, the aim of the present paper is to define a kind of "virtual augmentation" (as a substitute for the failing one), which is the D-Morita equivalence class of $G$, and which has to be considered intuitively as defining the "virtual structure" of the orbit space.

7.3.1. Plurigroups. An important special case of regular D-groupoid is when $R=\circ B$ (7.1.3) (this is indeed equivalent to $\alpha_{G}=\beta_{G}$ ). When such is the case, we shall say $G$ is a "D-plurigroup" 49 .

7.3.2. s-transitive $\mathrm{D}$-groupoids. The opposite degeneracy is when $\tau_{G}$ is a good epi: we shall say $G$ is $s$-transitive.

When $\mathrm{D}=$ Set, this just means that the orbit space is reduced to a singleton, but in Dif, for instance, this has very strong implications, since this means essentially that $G$ may be viewed as the gauge groupoid of a principal bundle50. This can be proved in a purely diagrammatic way, which allow to extend these concepts to all (Godement) diptychs. In fact the orbit space has to be thought as "a singleton structured by a group".

One of the basic reason of the strength of the notion of D-groupoid is that it unifies and gathers in a single theory all these various degeneracies.

\footnotetext{
${ }^{48}$ Where the formal aspect of this theorem is clearly visible, and inspired our Godement axiom.

${ }^{49}$ We keep the term D-group for the case when moreover $B$ is a terminal object. On the other hand, the possible term "multigroup" would create confusion with the multiple categories, which have nothing to do with the present case.

${ }^{50}$ The term "Lie groupoid" was first reserved to that special case (see for instance the first textbook by K. Mackenzie), till A. Weinstein and P. Dazord changed the terminology, with my full agreement.
} 


\section{The CATEgory Gpd(D).}

\subsection{D-functors.}

8.1.1. D-functors as natural transformations. D-functors (or morphisms) between D -groupoids are of course special cases of diagram morphisms (5) and, as such, are defined as natural transformations between the $D$-groupoids viewed as functors.

But the pullback property of the generating squares of the diagrams defining groupoids (6.2.2) has very strong implications (arising from the preliminary study of commutative squares in a diptych) which we cannot develop here, referring to [P4] for more details. We just mention a few basic facts.

A D-functor $\mathbf{f}: \mathbf{H} \rightarrow \mathbf{G}$ is fully determined by $f^{(1)}: H^{(1)} \rightarrow G^{(1)}$ and hence often denoted loosely by $f: H \rightarrow G$; we shall write : $H^{(0)}=E, G^{(0)}=B$. It is called principal if $H$ is principal.

We say $f$ is a $i / s$-functor when $f$ lies in $\mathcal{D}_{i} / \mathcal{D}_{s}$; as a consequence, one can check this is still valid for all the $f^{(n)}$.

We get in this way the category $\operatorname{Gpd}(\mathrm{D})$, with $\operatorname{Gpd}(\mathrm{D})$ as its base, of (Dfunctors or morphisms between) D-groupoids, and two subcategories $\mathbf{G p d}_{i}(\mathrm{D})$ and $\operatorname{Gpd}_{s}(\mathrm{D})$, but, as announced earlier, the second one will not be the right candidate for good epis in $\operatorname{Gpd}(\mathrm{D})$ (see below).

An arrow of $\operatorname{Gpd}(\mathrm{D})$ is said to be split if it is right invertible, in other words if it admits of a section.

8.1.2. D-functors as ( $\left.\square^{\text {vert }} \mathrm{D}\right)$-groupoids. Following (5.1), a D-functor may be viewed as a $\left(\square^{\text {vert }} \mathrm{D}\right)$-groupoid 51 .

8.2. Actors. It turns out that the basic (algebraic) 52 properties of a D-functor $f: H \rightarrow G$ are encapsulated into two fundamental squares, written below, which immediately acquire a "structured" (and hence more precise) meaning when written in a diptych.

It is enough to write these properties at the lowest level $\left(f^{(0)}, f^{(1)}\right)$, since it turns out that the special pullback properties of the nerve, as a diagram, allow to carry them over to all levels.

8.2.1. The activity indicator $\mathrm{A}(f)$ : in/ex/-actors. We consider first the commutative square generated by the source maps :

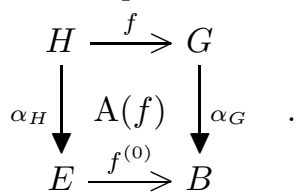

We shall say $f$ is : an actor 53 , an inactor, an exactor 54, depending on whether the square $\mathrm{A}(f)$ is a pullback, $i$-faithful, or $s$-full (4.1).

\footnotetext{
${ }^{51}$ One has to check the exactness property.

${ }^{52}$ Most of these properties own various names in the literature, depending on the authors, and equally unfortunate for our purpose, since these purely algebraic properties received often names issued from Topology, which cannot be kept when working in Top or Dif.

${ }^{53}$ See (2.3) for the terminology.

${ }^{54}$ The underlying algebraic notion is known in the categorical literature under the name of "fibering functors" (or "star-surjective" functors for Ronnie Brown), which cannot be kept when working in a topological setting.
} 
One can show that any exactor $f: H \rightarrow G$, one can define its kernel $K \longmapsto H$, which is null when $f$ is an actor.

A principal actor is an actor $R \stackrel{f}{\rightarrow} G$ with $R$ principal. For instance, taking for $R$ the graph associated to a covering, as described in (2.2), we recover the notion of $G$-cocycle, including cocycles defining a principal 55 fibration (when $G$ is a Lie group) and Haefliger cocycles defining a foliation (when $\mathrm{G}$ is a pseudogroup).

8.2.2. The canonical actor $\delta_{G}$. The map $\delta_{G}: \wedge G=\triangle G \longrightarrow G$ may be viewed as a functor, and indeed a principal $s$-actor, associated to the right action of $G$ on itself.

This will be enlightened by the functorial considerations to be developed below.

\subsection{D-equivalences.}

8.3.1. The full/faithfulness indicator $\mathrm{T}(f)$. The second basic square is built with the transitors (anchor maps).

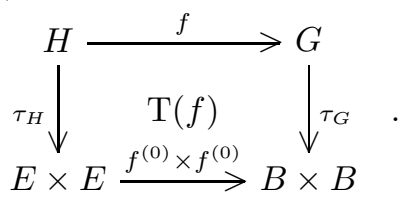

8.3.2. Equivalences and extensors. We shall say $f$ is an inductor/i-faithful/s-full, depending on whether the square $\mathrm{T}(f)$ is a pullback $/ i$-faithful $/ s$-full56 (4.1).

When $f$ is $s$-full/a D-inductor, and moreover $f^{(0)}$ lies in $\mathcal{D}_{s}$, then $f$ is an $s$ functor, and we say $f$ is an s-equivalence/an s-extensor 57 .

While the concept of D-inductor derives from the diagrammatic description of "full and faithful", the general concept of D-equivalence demands to add a diagrammatic description of the "essential (or generic) surjectivity", which uses the $A(f)$ square and will not be given here (we refer to [P4]). This general notion allows to speak of $i$-equivalences too.

Parallel to the notion of canonical actor, we have those of canonical equivalences. These will be defined below, when we have given a diagrammatic construction of $\square G$ and of the canonical morphisms:

$$
G \rightarrow \square G \rightrightarrows G .
$$

8.4. Diptych structures on $\operatorname{Gpd}(\mathrm{D})$. The following result, which we can just mention here, is of basic importance for a unified study of structured groupoids.

Using the preliminary study of diagrams in a diptych, one can define several useful (Godement) diptych structures on Gpd(D).

We stress the fact that the $s$-functors are not the right candidates for good epis 58 .

Among various possibilities, one can take:

\footnotetext{
${ }^{55}$ Whence the terminology.

${ }^{56} \mathrm{As}$ announced, this explains the terminology used for the squares.

${ }^{57}$ The terminology derives from the following fact : it turns out that such functors are the exact generalizations of Lie group extensions (save for the fact that one has to use two-sided cosets, which, in general, don't coincide with right or left cosets.). The smooth case is treated in [P2], which is written in order to be read possibly in any diptych without any change.

${ }^{58}$ This problem doesn't arise in the set-theoretic case. Many authors seem to believe that pullbacks along $s$-functors always exist in the Dif case, but actually the delicate point, often forgotten, is to prove the surmersion condition for the source map.
} 
- for good monos:

- either the $i$-functors

- or the $i$-actors;

- for good epis those $s$-functors which moreover belong to one of the following types:

$-s$-exactors

$-s$-actors

- $s$-equivalences.

8.5. The category $\operatorname{Gpd}\left(\mathrm{N}_{c}^{+*}\right)$. $\mathrm{D}^{*}$-groupoids may be called D-cogroupoids.

Some constructions for D-groupoids may be better understood from a study of $\mathrm{N}_{c}^{+*}$-groupoids or $\mathrm{N}_{c}^{+}$-cogroupoids (which are not Set-groupoids) (3.4.1). Some pieces of notations are needed to avoid confusions arising from duality.

8.5.1. Notations for $\operatorname{End}\left(\mathcal{N}_{\mathrm{c}}^{+}\right)$and $\operatorname{End}\left(\mathcal{N}_{\mathrm{c}}^{+*}\right)$. In contrast to sections (4) and (5), we shall, for a while, stick to Mac Lane's terminology concerning horizontal and vertical composition of natural transformations (also called functorial morphisms), in order to allow free use of $[\mathrm{McL}]$ as reference.

This means that groupoids (viewed as diagrams or functors) have here to be thought as written horizontally (instead of vertically as above), hence the groupoid functors or morphisms (i.e. the arrows of $\mathbf{G p d}(\mathrm{D})$ ) as written vertically, though this is somewhat uncomfortable.

The natural transformations between endofunctors make up categories denoted by $\operatorname{End}\left(\mathcal{N}_{c}^{+}\right)$and $\operatorname{End}\left(\mathcal{N}_{c}^{+*}\right)$, which are indeed double categories (and even more precisely 2 -categories $[\mathrm{McL}]$ ) when considering both horizontal and vertical composition.

The identity maps define canonically:

- a contravariant functor:

$$
\mathcal{N}_{c}^{+} \rightarrow \mathcal{N}_{c}^{+*}, \lambda \mapsto \lambda^{*},
$$

where $\lambda^{*}$ is $\lambda$ with source and target exchanged ; denoting by ${ }^{*}$ the product in $\mathcal{N}_{c}^{+*}$ (i.e. the sum + in $\mathcal{N}_{c}^{+}$), we can write (for any pair of arrows $\lambda, \mu$ ):

$$
(\lambda+\mu)^{*}=\lambda^{*} \stackrel{*}{\times} \mu^{*}
$$

- a covariant functor:

$$
\operatorname{End}\left(\mathcal{N}_{\mathrm{c}}^{+}\right) \rightarrow \operatorname{End}\left(\mathcal{N}_{\mathrm{c}}^{+*}\right), \Phi \mapsto{ }^{*} \Phi^{*}=\stackrel{*}{\Phi},
$$

with $\stackrel{*}{\Phi}\left(\lambda^{*}\right)=(\Phi(\lambda))^{*}$;

- a bijection

$$
\operatorname{End}\left(\mathcal{N}_{c}^{+}\right) \rightarrow \operatorname{End}\left(\mathcal{N}_{c}^{+*}\right),\left(\varphi: \Phi \dot{\rightarrow} \Phi^{\prime}\right) \mapsto\left(\varphi^{*}: \stackrel{*}{\Phi} \dot{\leftarrow} \stackrel{*}{\Phi}^{\prime}\right),
$$

with $\varphi^{*}(n)=(\varphi(n))^{*}$, which is:

- covariant with respect to horizontal composition laws ;

- contravariant with respect to vertical composition laws.

8.5.2. Description of $\mathbf{G p d}\left(\mathrm{N}_{c}^{+*}\right)$. By the previous bijection:

- $\mathrm{N}_{c}^{+*}$-groupoids derive covariantly from the endofunctors of $\mathrm{N}_{c}^{+}$preserving surjections, injections and pushouts;

- morphisms of $\mathrm{N}_{c}^{+*}$-groupoids derive contravariantly from morphisms between endofunctors of the previous type. 
Moreover we know from the general descriptions of D-groupoids (6.2.3) that:

- the endofunctors $\Gamma=\stackrel{*}{\Phi}$ of $\mathrm{N}_{c}^{+*}$ defining $\mathrm{N}_{c}^{+*}$-groupoids are uniquely determined by the data:

$$
\left(\Gamma^{(0)}, \Gamma^{(1)}, \omega_{\Gamma}, \alpha_{\Gamma}, \delta_{\Gamma}\right)
$$

hence (resulting from the previous study) by the data in $\mathrm{N}_{c}^{+}$:

$\left(n_{0}=\Phi(\cdot 0), n_{1}=\Phi(\cdot 1), n_{2}=\Phi(\cdot 2), \omega: n_{0} \longleftarrow n_{1}, \alpha: n_{1} \longleftrightarrow n_{0}, \delta: n_{2} \longleftrightarrow n_{1}\right)$.

8.5.3. Some basic examples of $\mathrm{N}_{c}^{+*}$-groupoids and morphisms. As just explained, such a groupoid morphism $\gamma: \Gamma \leftarrow \Gamma^{\prime}$ derives from a functorial morpism $\varphi$ : $\Phi \rightarrow \Phi^{\prime}$ where $\Phi, \Phi^{\prime}$ preserve surjections, injections and pushouts. These define a 2-subcategory of $\operatorname{End}\left(\mathcal{N}_{c}^{+}\right)$denoted by $\digamma$.

Those $\Phi$ 's which preserve sums too are of type :

$$
\boldsymbol{p}_{\times}: \cdot n \mapsto p \times \cdot n, \lambda \mapsto p \times \lambda\left(p \text { times iterated sum in } \mathrm{N}_{c}^{+}\right)
$$

The associated groupoids, denoted by $\boldsymbol{p}_{\times}^{*}$ are the banal (but not principal 59 ) $\mathbf{N}_{c}^{+*}-$ groupoids, in the sense of (7.2.3) 60 .

For $p=0$, we get the "null groupoid", denoted by $\mathbf{0}$, associated to the constant functor $\mathbf{0}: \cdot n \mapsto \cdot 0$, and, for $p=1$, the "unit groupoid", denoted by $\mathbf{1}$, associated to the identity functor.

For $p=2$, one has the "square groupoid" 61, denoted by $¥=\mathbf{2}_{\times}^{*}$, associated to:

$$
\mathbf{2}_{\times}: \cdot n \mapsto 2(\cdot n)=\cdot n+\cdot n, \lambda \mapsto 2 \lambda=\lambda+\lambda .
$$

Among the non principal ones (7.2.2), the simplest is denoted by : it is associated to the "shift functor":

$$
\mathbf{0}_{+}: \cdot n \mapsto \cdot 0+\cdot n, \lambda \mapsto \cdot 0+\lambda
$$

where the last $\cdot 0$ is understood as the identity of the object $\cdot 0=1$.

We have also a $\mathrm{N}_{c}^{+*}$-groupoid morphism:

$$
\delta_{0}^{*}: \text { 疋 } 1
$$

associated, with the notations of (7.1.1) and of [McL], to the the "shift morphism", defined by the family $\left(\delta_{0}^{\cdot n}\right)_{n \in \mathbb{N}}$ (injections skipping the 0 in $\cdot 1+\cdot n$ ).

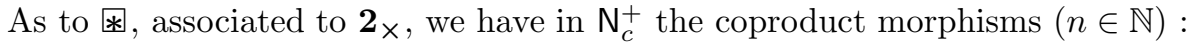

$$
(\cdot n) \stackrel{\text { codiag }}{\longleftarrow}(\cdot n)+(\cdot n) \stackrel{\iota_{2}}{\longleftarrow}(\cdot n)
$$

which define morphisms of $\mathrm{N}_{c}^{+*}$-groupoids denoted suggestively by:

$$
1 \stackrel{\omega}{\longrightarrow} \underset{\varpi_{1}}{\stackrel{\varpi_{2}}{\longrightarrow}} 1 \text {. }
$$

By iteration we can even get a canonical groupoid (荬 $\left.{ }^{(n)}\right)_{n \in \mathbb{N}}$ in $\operatorname{Gpd}\left(\mathbf{N}_{c}^{+*}\right) 62$.

The "symmetry groupoid" $\boldsymbol{\Sigma}_{*}$ proceeds from the (involutive) "symmetry map" $\Sigma: \mathcal{N}_{c}^{+} \rightarrow \mathcal{N}_{c}^{+}$, derived by reversing the order on the integers viewed as ordinals

\footnotetext{
${ }^{59}$ Since the terminal object 0 has been dropped.

${ }^{60}$ While the banal (and principal) $\mathrm{N}_{c}^{+}$-groupoids are $p \times p$.

${ }^{61}$ The terminology will become clear below in 9.3

${ }^{62}$ More precisely, with a suitable diptych structure (see 8.4). This is indeed a double groupoid, or better a "groupoid-cogroupoid".
} 
$(\vec{n} \mapsto \overleftarrow{n})$; it is defined by the identity on the objects, and, on the generators (with Mac Lane's notations), by $\left(n \in \mathbb{N}^{+}\right)$:

$$
\Sigma: \delta_{j}^{n} \mapsto \delta_{n-j}^{n}, \sigma_{j}^{n} \mapsto \sigma_{n-j}^{n},
$$

and the family of maps : $\left(\varsigma_{n}: n \rightarrow n, j \mapsto n-j\right)_{\left(n \in \mathbb{N}^{+}\right)}$defines a natural transformation from identity towards $\Sigma$, hence also a groupoid morphism:

$$
\text { (3) } \varsigma: \Sigma_{*} \longrightarrow 1 \text {. }
$$

\section{Double Functoriality of the Definition of D-Groupoids.}

9.1. Bivariance of D-groupoids and D-functors. The definition of a D-groupoid as a diptych morphism $\mathbf{G}: \mathbf{N}_{c}^{+*} \rightarrow \mathrm{D}$ shows immediately (look at the adjoining di-

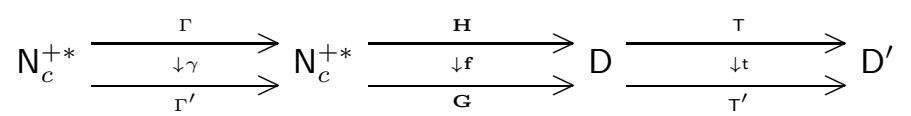

agram, where we go on sticking to Mac Lane's conventions) this definition is:

- functorial with respect to composition, on the left (target side), with an exact diptych morphism $\mathrm{T}: \mathrm{D} \rightarrow \mathrm{D}^{\prime}$, as well as with respect to right (horizontal ) composition with a natural transformation $t: T \rightarrow T^{\prime}$; this means that any D-functor $\mathbf{f}: \mathbf{H} \rightarrow \mathbf{G}$ gives rise to a $\mathrm{D}^{\prime}$-functor

$$
\mathbf{T} \circ \mathbf{f}: \mathbf{T} \circ \mathbf{H} \rightarrow \mathrm{T} \circ \mathbf{G}
$$

63 and to a natural transformation:

$$
\mathrm{t} \circ \mathbf{f}: \mathrm{T} \circ \mathbf{f} \rightarrow \mathrm{T}^{\prime} \circ \mathbf{f}
$$

the latter gives rise to the following commutative square of $\mathrm{D}^{\prime}$-groupoids 64 :

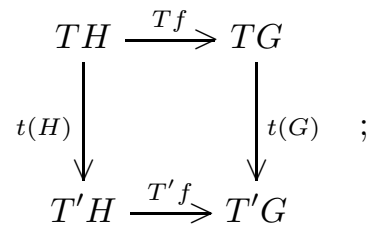

actually Gpd behaves here like a functor and we can define:

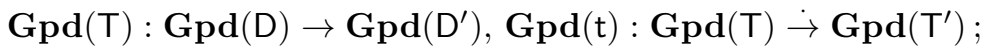

- functorial with respect to horizontal composition on the right (source side), with a $\mathrm{N}_{c}^{+*}$-groupoid $\Gamma$, as well as with a groupoid morphism $\gamma: \Gamma \rightarrow$ $\Gamma^{\prime}$ viewed as a natural transformation between endofunctors of $\mathrm{N}_{c}^{+*}$; this means that any D-functor $\mathbf{f}: \mathbf{H} \rightarrow \mathbf{G}$ gives rise to a D-functor:

$$
\Gamma^{\bullet} \mathrm{f}: \Gamma^{\bullet} \mathrm{H} \rightarrow \Gamma^{\bullet} \mathrm{G}
$$

where

$$
\Gamma^{\bullet} \mathbf{H}=\mathbf{H} \circ \boldsymbol{\Gamma}, \Gamma^{\bullet} \mathbf{G}=\mathbf{G} \circ \boldsymbol{\Gamma}, \Gamma^{\bullet} \mathbf{f}=\mathbf{f} \circ \boldsymbol{\Gamma},
$$

and to a natural transformation

$$
\gamma^{\bullet} \mathbf{f}=\mathbf{f} \circ \gamma: \Gamma^{\bullet} \mathbf{G} \rightarrow \Gamma^{\bullet \bullet} \mathbf{G}
$$

\footnotetext{
${ }^{63} \mathrm{As}$ in $[\mathrm{McL}]$, the same notation is used for a functor and the identity natural transformation associated to this functor, here $\mathrm{T}$

${ }^{64}$ Written in loose notations, i.e. identifying groupoids and functors with their 1-level part.
} 
this defines a (covariant) functor:

$$
\Gamma^{\bullet}: \operatorname{Gpd}(\mathrm{D}) \rightarrow \mathbf{G p d}(\mathrm{D}),
$$

hence a canonical (vertical) representation:

$$
\operatorname{Gpd}\left(\mathrm{N}_{c}^{+*}\right) \rightarrow \operatorname{Gpd}(\mathrm{D})
$$

but this representation depends in a contravariant way upon $\Gamma$, with respect to the horizontal composition of $\mathrm{N}_{c}^{+*}$-groupoids defined above, since

$$
\boldsymbol{\Gamma}^{\bullet} \circ \boldsymbol{\Gamma}^{\bullet}=\left(\boldsymbol{\Gamma}^{\prime} \circ \boldsymbol{\Gamma}\right)^{\bullet}, \gamma^{\bullet} \circ \gamma^{\bullet}=\left(\gamma^{\prime} \circ \gamma\right)^{\bullet} .
$$

Note that, when going back to the generating endofunctors of $\mathrm{N}_{c}^{+}$, we get a doubly contravariant canonical representation of $\digamma$ (notation of 8.5.3) into $\mathbf{G p d}(\mathrm{D})$.

We give a few examples.

9.2. Examples for the left functoriality. We can either "forget" the structures on objects of D, or "enrich" them. We give examples of these opposite directions.

9.2.1. Concrete diptychs. Thinking to the basic examples of Top and Dif, we shall say D is "concrete" if it comes equipped with an adjunction from E = Set to D defined by the following adjoint pair [McL] of functors 65 :

$$
(\text { discrete, forgetful })=(\cdot,||): \mathrm{E} \underset{11}{\longrightarrow} \mathrm{D} \text {. }
$$

Then we can speak of the underlying E-groupoid, and make use of set-theoretical descriptions.

9.2.2. The tangent functor. Thinking now to the case when $\mathrm{D}=\mathrm{Dif}$, we can consider (see 3.3.6) the tangent functor:

$$
\text { T : Dif } \longrightarrow \text { VectB, }
$$

which is equipped with two natural transformations:

$$
0 \stackrel{\cdot}{\rightarrow} T \underset{t}{\rightarrow} 0
$$

Once one has checked it defines an exact diptych morphism, we can immediately transfer to the tangent groupoids all the general constructions valid for general D-groupoids (for instance constructions of fibred products, and so on).

9.2.3. Double groupoids. The idea of defining and studying the notion of double groupoids as groupoids in the category of groupoids is due to Ehresmann, who proved the equivalence with the alternative description by means of two category composition laws satisfying the "exchange law" 66

In the diptych setting we get several notions depending on the choice for the diptych structure on $\operatorname{Gpd}(\mathrm{D})$ (see 8.4).

We stress the point that, even in the purely set-theoretical setting, the choice we made of exactors for good epis implies adding a certain surjectivity condition 67 which does not appear in Ehresmann's definition, but was encountered by several

\footnotetext{
${ }^{65}$ Assumed moreover to be faithful, to preserve products, and to define exact diptych morphisms. An object $B$ of $\mathcal{D}$ is viewed as a "structure" on the "underlying set" $|B|$, and an arrow $A \rightarrow B$ of $\mathcal{D}$ is fully described by the triple $(A,|f|, B)$. Any set $E$ may be endowed with the "discrete structure" $\dot{E}$.

${ }^{66}$ In our framework this would result from 5.3

${ }^{67}$ If we consider the square made up by the sources and targets of both laws, this condition means that three of the four edges may be given arbitrarily.
} 
authors, mainly Ronnie Brown (filling condition), and seems useful to develop the theory beyond just definitions.

Applying the general theory of D-diptychs, for instance for getting fibred products, or quotients, or Morita equivalences, gives results which it would be very hard to get by a direct study (which has never been done), even in the purely algebraic setting.

9.3. Examples for the right functoriality. As announced, the coherence of various notations introduced above will appear just below (to be precise, we find : $\mathbb{A}^{\bullet}=\triangle$, 囷 $=\square$ ). The examples given in 8.5 .3 allow to transfer to $\mathrm{D}$-groupoids some classical set-theoretic constructions, announced above. Taking for $\gamma: \Gamma \rightarrow \Gamma^{\prime}$ :

- formula (1) of 8.5 .3 we recover the morphism:

$$
\delta_{G}: \triangle G \longrightarrow G
$$

- formula (2), we get the canonical equivalences:

$$
G \rightarrow \square G \rightrightarrows G ;
$$

- formula (3), we get the dual groupoid $G^{*}=\Sigma G$, and the inverse law $\varsigma_{G}=\iota_{G}$, which defines an involutive isomorphism:

$$
\varsigma_{G}: G \rightarrow G^{*} .
$$

9.4. D-natural transformations, holomorphisms. Once $\square G$ is defined, one can also define D-natural transformations between functors from $H$ to $G$ as Dfunctors $H \rightarrow \square G$. The canonical $i$-equivalence $G \rightarrow \square G$ defines the identical transformation of the identity functor.

Since $G$ is a groupoid, such natural transformations are necessarily functorial isomorphisms. An isomorphy class of D-functors will be called a "holomorphism" (an alternative terminology might be "exomorphism", since this notion generalizes the outer automorphisms of groups).

Since the horizontal composition of natural transformations commutes with the vertical one, it defines a composition between holomorphisms, and this yields a quotient category of $\mathbf{G p d}(\mathrm{D})$, which we shall denote by $\mathbf{H o l}(\mathrm{D})$.

Any D-functor $f: H \rightarrow G$ generates the following commutative diagram, in

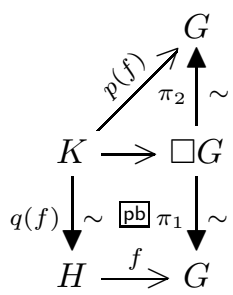

which $q(f)$ is an $s$-equivalence, and $p(f)$ an exactor. The pair $(p(f), q(f))$ is called the "holograph" of $f$.

Moreover the $s$-equivalence $q(f)$ is split (8.1.1) since $\pi_{1}$ is. 


\section{The BUtTerfly DiAgRAM.}

This section illustrates, in the case of orbital structures presently described, the use of diagrams in a diptych for transferring constructions in Set to constructions in Dif or other various categories, as well as the use of the various kinds of Dfunctors introduced above. We can be only very sketchy. More precise descriptions and results may be found in $[\mathrm{P} 4]$, where they are stated for the differentiable case, but written to be easily transferable to general diptychs. More details about the diagrams used for proofs will be given elsewhere.

\subsection{Generalized "structure" of the orbit space.}

10.1.1. Algebraic "structure". First, from a purely set-theoretic point of view, any equivalence between two groupoids, in the general categorical sense $[\mathrm{McL}] 68$, preserves 69 the set-theoretic orbit space $Q$, but indeed it preserves much more, since, to each orbit (or transitive component), there is a (well defined only up to isomorphism) attached isotropy group, and this endows $Q$ with a kind of an algebraic "structure", in a (non set-theoretical) generalized sense. For instance, the leaves of a foliation are marked by their holonomy groups, the orbits of a group(oid) action are marked by their fixators. Such a "structure" is sometimes called a group stack.

10.1.2. D - "structure" of the orbit space. Then, in the D-framework, replacing algebraic equivalences by D-equivalences will moreover encapsulate in this generalized structure the memory of the D-structure as well. It turns out (though this is by no means a priori obvious) that it is enough to make use of $s$-equivalences (8.3.2). Finally we are led to the following:

Definition: Two D-groupoids $H, G$, are said to be D-equivalent if they are linked by a pair of $s$-equivalences: $H \underset{\sim}{\stackrel{q}{\sim}} K \underset{\sim}{\sim} G$.

The fact that this is indeed an equivalence relation is an easy consequence of the results stated in 8.4 taking $s$-equivalences as good epis in $\mathbf{G p d}(\mathrm{D})$, and using fibred products of good epis.

A D-equivalence class of D-groupoids may be called an orbital structure. Any representative of this equivalence class is called an atlas of the orbital structure.

\subsection{Inverting equivalences.}

10.2.1. Meromorphisms. Note than in general orbital structures cannot be taken as objects of a new category. However one can define [P4] a new category which shall be denoted here by Mero(D), with the same objects as $\mathbf{G p d}(\mathrm{D})$, and arrows called "meromorphisms", in which the s-equivalences (and indeed all the D-equivalences) become invertible, in other words are turned into isomorphisms. In the topological case, these isomorphisms may be identified with the Morita equivalences. In this new category the orbital structures now become isomorphy classes of D-groupoids, though the objects still remain D-groupoids and not isomorphy classes, so that the orbital "structures" are not carried by actual sets, and, as such, remain "virtual".

This means that Mero(D), is the universal solution for the problem of fractions consisting in formally inverting the $s$-equivalences, and indeed all the Dequivalences.

\footnotetext{
68 i.e. a functor which is full, faithful, and essentially surjective, but possibly non-surjective.

${ }^{69}$ More precisely, this means that it defines a bijection between the two orbit spaces.
} 
This kind of problem always admits a general solution [G-Z]: the arrows are given by equivalence classes of diagrams, the description of which is simpler when the conditions for right calculus of fractions are satisfied.

It is worth noticing that these assumptions are not fulfilled here, while our construction is in a certain sense much simpler, since, as is the case in Arithmetic, each fraction will admit of a simplified or irreducible canonical representative $(p, q)$. In the topological case these representatives may be identified with the "generalized homomorphisms" described by A. Haefliger in [Ast 116] and attributed to G. Skandalis, or the " $K$-oriented morphisms" of M. Hilsum and G. Skandalis.

We can say more. The canonical functor $\operatorname{Gpd}(\mathrm{D}) \stackrel{\Phi}{\rightarrow} \operatorname{Mero}(\mathrm{D})$ admits of the following factorization: $\operatorname{Gpd}(\mathrm{D}) \stackrel{\Phi_{1}}{\rightarrow} \mathbf{H o l}(\mathrm{D}) \stackrel{\Phi_{2}}{\rightarrow} \operatorname{Mero}(\mathrm{D})(\operatorname{see} 9.4)$ with $\Phi_{1}$ full (i.e. here surjective) and $\Phi_{2}$ faithful (or injective).

It turns out that $\mathbf{H o l}(\mathrm{D})$ is the solution of the problem of fractions for split (8.1.1) D-equivalences. It is embedded in Mero(D) by means of the holograph (9.4).

10.2.2. Description of fractions. Let $(p, q)$ denote a pair of exactors with the same source $K: p: K \rightarrow G, q: K \rightarrow H$. We set $R=\operatorname{Ker} q, S=\operatorname{Ker} p$ (see 8.2).

Let $\left(p^{\prime}, q^{\prime}\right)$ another pair $p^{\prime}: K^{\prime} \rightarrow G, q^{\prime}: K^{\prime} \rightarrow H$ with the same $G$ and $H$.

Letting for a while $G$ and $H$ fixed, we start considering arrows $k:\left(p^{\prime}, q^{\prime}\right) \rightarrow(p, q)$ defined as $D$-functors $k: K^{\prime} \rightarrow K$ such that the whole diagram commutes.

Let $p / q$ denote the isomorphy class of $(p, q)$, and call it a "fraction".

On the other hand we say two pairs $\left(p_{i}, q_{i}\right)(i=1,2)$ are equivalent if there exist two $s$-equivalences $k_{i}:(p, q) \rightarrow\left(p_{i}, q_{i}\right)$. This is indeed an equivalence relation, and the class of $(p, q)$ will be denoted by $p q^{-1}$.

We consider now those pairs $(p, q)$ satisfying the subsequent extra conditions, which turn out to be preserved by the previous equivalence:

(1) $q$ is an $s$-equivalence;

(2) $p$ and $q$ are "cotransversal".

The former condition implies that the kernel $R=\operatorname{Ker} q$ is principal (7.2.2). The latter condition will be expressed by means of the following (commutative) "butterfly diagram" gathering the previous data:

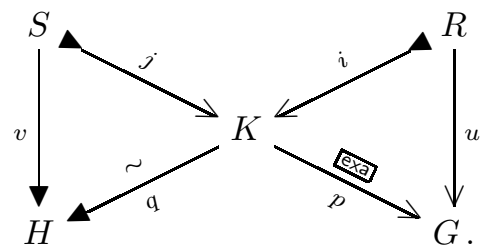

Then the condition of cotransversality means that $u$ or (this is indeed equivalent) $v$ is an exactor (then $v$ will be an $s$-exactor).

When $u$ (and $v$ ) are actors, $p$ and $q$ are said to be "transverse", and the fraction $p / q$ is called "irreducible" (or simplified).

One can show (using the theory of extensors) that the class $p q^{-1}$ owns a unique irreducible representative $p / q$.

Our meromorphisms (from $H$ to $G$ ) are then defined as the classes $p q^{-1}$ or their irreducible representatives $p / q$. 
A "Morita equivalence" is the special case when $p$ is an $s$-equivalence too. The butterfly diagram is then perfectly symmetric. We say that $(u, v)$ is a pair of "conjugate principal actors". Each one determines the other one up to isomorphism.

Using irreducible representatives and forgetting the D-structures, one then recovers easily the set-theoretical part of the description of Skandalis-Haefliger homomorphisms (two commuting actions, one being principal). Now, in the differentiable case, the local triviality conditions are automatically encapsulated in the surmersion conditions (imposed to the good epis) by means of the Godement theorem.

One of the immense advantages of this presentation (apart from being defined in many various frameworks), is that the use of non irreducible representatives allows a very natural definition of the composition of meromorphisms (note that in [Ast 116] this composition is defined by A. Haefliger but in very special cases, when one arrow is a Morita equivalence). This composition is defined by means of the following diagram (using the diptych properties of $\mathbf{G p d}(\mathrm{D})$ ) (8.4):

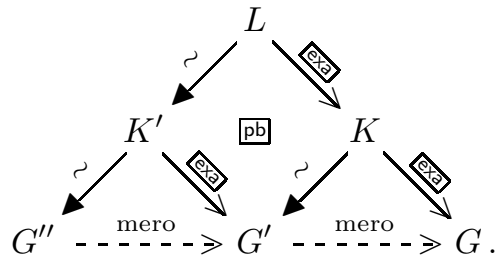

(Of course there are many things to check to justify all our claims).

10.3. Example. Let us come back to the example of the space of leaves of a (regular) foliation (2.4). We invite the reader to look at what happens when we take as good epis:

(1) all the surmersions;

(2) the retroconnected ones;

(3) the retrodiscrete (or étale) ones;

(4) the proper ones.

In the first case, we are allowed to take as an atlas the holonomy groupoid and the transverse holonomy pseudogroups associated to various tranversals as well, which all belong to the same Morita class.

The second choice is adapted to the search for invariants of the Molino equivalence class of a foliation: the Morita class of the holonomy groupoid is such an invariant.

The third choice is adapted to the use of holonomy pseudogroups and of van Est S-atlases [Ast 116], and to the study of the effect of coverings on foliations.

The fourth choice is adapted to the study of compact leaves of foliations and of properties related to Reeb stability theorem, as well as to the study of orbifolds (or Satake manifolds).

In fact it is useful to use various choices simultaneously.

\section{Epilogue.}

- We have not given here statements concerning diptych structures on the category Mero(D), since our present results are still partial and demand some further checks to ensure them completely, especially concerning the Godement property. It is clear for us that such types of statements would be very useful, since, for instance, groupoids in such diptychs would be 
fascinating objects. Anyway it seems clear for us that this category has to be explored more deeply.

- We know that our formal construction for the previous category of fractions seems to work perfectly as well when replacing the category of $s$ equivalences by that of $s$-extensors or by various subcategories of the latter (adding for instance conditions of connectedness on the isotropy groups). We are convinced that such categories, which are much less known (not to say totally unknown) than the previous one, are basic for the understanding of holonomy of foliations with singularities (Štefan foliations), and that these enlarged Morita classes certainly encapsulate some deep and hidden properties of the orbit spaces.

\section{Acknowledgements.}

(1) I would like to thank warmly the organizers for their invitation to this Conference at Krynica. I was happy with the friendly and stimulating atmosphere which was reigning throughout this session.

(2) I am indebted to Paul Taylor for the (certainly very awkward) use I made of his package "diagrams", allowing various styles of arrows, which are very useful as condensed visual mnemonics for memorizing properties of maps and functors.

\section{REFERENCES}

1. [P1] J.Pradines: "Building categories in which a Godement theorem is available", Cahiers Top. et Géom. Diff., XVI-3 (1975), 301-306.

2. [P2] _ _ "Quotients de groupoïdes différentiables", C.R.A.S., Paris, 303, 16 (1986), 817820.

3. [P3] _ : "How to define the graph of a singular foliation", Cahiers Top. et Géom. Diff., XVI-4 (1985), 339-380.

4. [P4] _ : "Morphisms between spaces of leaves viewed as fractions", Cahiers Top. et Géom. Diff., XXX-3 (1989), 229-246.

5. [P5] _ :Feuilletages: holonomie et graphes locaux", C.R.A.S., Paris, 298, 13 (1984), 297-300.

6. [P6] _ : "Remarque sur le groupoïde cotangent de Weinstein-Dazord", C.R.A.S, Paris, 306, (1988), 557-560.

7. [B] N. Bourbaki: Variétés différentielles et analytiques, Hermann, Paris, 1971.

8. [McL] S. Mac Lane: Categories for the Working Mathematician, Springer-Verlag, New York, 1971.

9. [LALG] J.-P. Serre: Lie Algebras and Lie Groups,W. A. Benjamin Inc., New York, 1965.

10. [G-Z] P. Gabriel and M. Zisman: Calculus of fractions and homotopy theory, Ergebn. Math. 35, Springer, 1965.

11. [Ast. 116] Astérisque 116, Structure transverse des feuilletages, Société Mathématique de France Paris, 1984.

A. Haefliger: "Groupoïdes d'holonomie et classifiants", pp. 70-97.

W. T. van Est: "Rapport sur les S-atlas", pp. 235-292.

26, Rue Alexandre Ducos, F31500 Toulouse, France

E-mail address: jpradines@wanadoo.fr 